\title{
ELLIPTIC OPERATORS AND THE DECOMPOSITION OF TENSOR FIELDS
}

\author{
BY MURRAY CANTOR ${ }^{1}$
}

CONTENTS

1. Introduction

2. Splittings of Banach spaces

3. The Fredholm alternative for differential operators over compact manifolds

4. Decomposition of tensor fields over compact manifolds

5. Weighted Sobolev spaces over $\mathbf{R}^{n}$

6. Elliptic operators on weighted Sobolev spaces

7. Decomposition of tensor fields over asymptotically Euclidean manifolds

1. Introduction. The decomposition of tensor fields into canonical forms arises as an important step in many problems of mathematics and physics. The classical Helmholtz decomposition (divergence free plus gradient) arises in fluid mechanics and electromagnetism. The Hodge-Kodaira decomposition and its generalizations is an important area of mathematical study. More recently various decompositions of 2-tensors have arisen in the study of general relativity $[6,16,30]$. There have also been applications in differential geometry $[4,15,17]$ and symplectic structures [3].

There are, of course, classical methods for the study of some decompositions. These involve the treatment of a single tensor (or vector) field. This is inadequate for most applications. Often one needs to split entire spaces of tensor fields.

The reason for this is that the desired decomposition usually is a linearization of a nonlinear problem. Let us give a simple example.

The configuration space for the dynamics of an incompressible fluid on a Riemannian manifold $(M, g)$ is the space of volume-preserving diffeomorphisms $\mathscr{D}_{\mu}$ on $M$. (Here $\mu$ is the canonical volume form determined by the metric g.) For more details see [21]. $\mathscr{D}_{\mu}(M)$ is properly thought of as a constraint space in the set of all diffeomorphisms of $M$. The Euler or Naviar-Stokes equations yield vector fields on $\mathscr{D}_{\mu}$. Thus a natural question is whether $\mathscr{D}_{\mu}$ is a submanifold. The principal tool for studying such a question is the implicit function theorem for Banach spaces.

Received by the editors November 1, 1980.

1980 Mathematics Subject Classification. Primary 58G99, 35J15.

${ }^{1}$ This research was supported by National Science Foundation Grant \# Phy7901801.

() 1981 American Mathematical Society 0002-9904/81/0000-0501/\$08.00 
IMPLICIT FUNCTION THEOREM. Let $\Phi: B_{1} \rightarrow B_{2}$ be a $C^{1}$ map between Banach manifolds. Then for $q \in B_{2}$, the set $\mathfrak{S}=\left\{p \in B_{1}: \Phi(p)=q\right\}$ is a submanifold of $B_{1}$ if for every $p \in \mathcal{S}$

(1) $T_{p} \Phi: T_{p} B_{1} \rightarrow T_{q} B_{2}$ is a surjection,

(2) $T_{p} B_{1}=\operatorname{ker}\left(T_{p} S\right) \oplus \mathcal{H}$ where $\mathcal{H}$ is closed.

Furthermore $T_{p} S=\operatorname{ker}\left(T_{p} \Phi\right)$.

See [19] for the proof of this theorem.

Note condition (2) is necessary. Thus a first step in studying fluid flow is determining whether the splitting given by condition (2) holds. As we shall see this entails the Helmholtz decomposition.

In order to apply the implicit function theorem one must work in a Banach manifold. The usual choice is $\mathbb{Q}^{p, s}$, the diffeomorphisms of class $W^{p, s}$ (or $\left.H^{s}=W^{2, s}\right)$ for $s>n / p+1$. In this case $\mathscr{Q}^{p, s}$ may be shown to be a manifold (see Marsden [21]). For $f \in \mathscr{Q}^{p, s}$ denote by $f^{*}(\mu)$ the usual action of $f$ on $\mu$ (see [1]). Let $\Phi: \mathscr{Q}^{p, s} \rightarrow W^{p, s-1}\left(\Lambda^{n} M\right)$ take $f$ to $f^{*}(\mu)$ and $\mathscr{Q}_{\mu}^{p, s}=\{f$ : $\Phi(f)=\mu\}$.

Let $I \in \mathscr{Q}_{\mu}^{p, s}$ be the identity map on $M$. Then $T_{I} \mathscr{Q}^{p, s}=W^{p, s}(T M)$, the vector fields on $M$ of class $W^{p, s}$. Calculation yields $T_{p} \Phi\left(V_{p}\right)=\operatorname{div}_{g} V_{p} \mu$ where $\operatorname{div}_{g}$ is the divergence associated with $g$. Thus condition (2) becomes

$$
W^{p, s}(T M)=\operatorname{ker}\left(\operatorname{div}_{g}\right) \oplus \mathscr{H}
$$

where $\mathcal{H}$ is closed. Hilbert space theory suggests that we write $\mathcal{H}$ as the range of the adjoint of $\operatorname{div}_{g}$. Even though we are using $W^{p, s}$ we use the formal or $L^{2}$ adjoint. Thus the decomposition of interest is

$$
W^{p, s}(T M)=\nabla_{g}\left(\left(W^{p, s+1}\right)(T M)\right) \oplus \operatorname{ker}\left(\operatorname{div}_{g}\right)
$$

where $\nabla_{g}$ is the gradient operator associated with $g$. Further we require the range of $\nabla_{g}$ to be closed. As we shall show (2) always holds for $p>1$ when $M$ is compact. The noncompact (asymptotically Euclidean) case is also treated in some detail.

The decomposition (2) is a prototype of the sorts of decompositions that arise in most problems. We will study decompositions of the form $B=$ $\operatorname{Rng}(D) \oplus \operatorname{ker}(E)$ where $B$ is some Banach space of sections and $D$ and $E$ are differential operators. Usually $D$ or $E$ has surjective symbol and $E$ is the formal adjoint of $D$.

In this paper a general framework for the study of such decompositions is given. Although for over a century there has been research on specific decompositions (see for example [18a]), there has not been much work on the general problem. One important paper giving a general theory is by Berger and Ebin [4]. They study the problem of splitting $H^{s}$ spaces of sections over compact manifolds with respect to elliptic operators. The earlier work in the field centers around the problem of decomposing alternating $k$-forms over compact manifolds. This was studied by Hodge [18b] and Kodaira [18c] in the thirties. Their work was extended by Morrey and Eells [23a, 23b]. All these authors studied the Hodge decomposition of $W^{p, k}$ forms over compact manifolds using a variational method. A different proof of this decomposition 
is given in the paper. (Morrey also studied the case of manifolds with boundary.)

In this paper a general procedure for deciding when decompositions hold is given in $\S 2$. This is used in $\S \S 3$ and 4 to state and prove a general decomposition theorem for Sobolev spaces of sections over compact manifolds. These results are an extension of the earlier work of Berger and Ebin mentioned above. Also the proof is somewhat simplified. Along the way (\$3) a proof of the Fredholm alternative for elliptic opeators on Sobolev spaces over compact manifolds is given. For $p=2$, this is a special case of some work of Kohn and Nirenberg [18d]. We know of no published source for this theorem for general $p$.

It is shown in $\$ 5$ that the usual decomposition theorems fail for Sobolev spaces of tensors over $\mathbf{R}^{n}$. The problem is that Sobolev spaces capture the local smoothness conditions necessary to invert the appropriate elliptic operators but fail to capture the correct growth at infinity behavior for solutions to potential type equations. The author [11] showed that some inequalities due to Nirenberg and Walker [24] implied that the Laplacian was an isomorphism between certain pairs of weighted Sobolev spaces. This result was generalized to operators with nonconstant coefficients in a later article [10]. Also in that article, some decompositions were demonstrated for fields with respect to asymptotically euclidean metrics over $\mathbf{R}^{n}$. There has been further work on the properties of elliptic operators on these spaces. The main result, due to McOwen [21, 23] (see also Lockhardt [20]) is that the previous results generalize to spaces with more general weights at infinity. He showed in this case the operators were Fredholm. He also showed the restrictions on $p$ required in the earlier papers were unnecessary. (See the definition of $M_{s, \delta}^{p}$ spaces below.) His results (for $p=2$ ) were extended to a slightly larger class of operators and to manifolds by Christodoulou and Choquet-Bruhat [14] (see also Cantor and Brill [12]). They did find some elegant lemmas involving the product structure of the weighted spaces. In $\$ \S 5-7$ all of this work is brought together and generalized to spaces of sections with general $p$ over noncompact manifolds. Many of the theorems are new. In particular necessary and sufficient conditions on the "growth at infinity" are given for particular tensor decompositions to hold.

The weighted Sobolev spaces are introduced and studied in $\$ 5$. Some rather delicate multiplication and growth theorems are presented. $\$ 6$ contains a rather general treatment of the elliptic theory on $\mathbf{R}^{n}$.

In $\$ 7$ the work in $\S 6$ is generalized to asymptotically euclidean manifolds.

Throughout we assume the reader knows elementary functional analysis. For $\S \S 3,4$ and 7 we assume the reader is familiar with the language of tensor bundles and spaces of sections. (This material may be found in $[19,1]$.)

$\S \S 5$ and 6 may be read separately and simply assume some familiarity with partial differential equations.

We will use standard multi-index notation (except in some parts of $\$ 5$ where, for convenience, total derivatives are used). Throughout large constants are denoted by $C$. The $L^{p}$ norm is given as ||$_{p}$, and the sup norm is denoted by \|\| . 
The author would like to thank Jerrold Marsden and James W. York for conversations which inspired this work.

2. Splittings of Banach spaces. In this section we present the fundamental lemma for verifying all decomposition theorems of the form

$$
X=T(Y) \oplus \operatorname{ker}(S)
$$

where $S$ and $T$ are bounded linear transformations between Banach spaces.

LEMMA 2.1. Let $T: X \rightarrow Y$ be a bounded linear transformation between Banach spaces. Suppose $Y=T(X) \oplus Z$ algebraically where $Z$ is closed. Then $T(X)$ is closed.

This lemma may be found in [26] but we will include the proof.

Proof. Since $\operatorname{ker}(T)$ is closed in $X$, we may construct the Banach space $W=X / \operatorname{ker}(T)$ and $\tilde{T}: W \rightarrow Y$ such that $\tilde{T}(W)=T(X)$ and $\tilde{T}$ is an injection. Also $\tilde{T}$ is bounded.

We may also construct the Banach space $W \oplus Z$ (by assumption $Z$ is a Banach space). The transformation $S(w, z)=\tilde{T}(w)+z$ is easily seen to be an isomorphism between $W \oplus Z$ and $Y$. By the open mapping theorem, $S$ is a closed map. Thus $T(X)=\{S(w, 0): w \in W\}$ is closed. Q.E.D.

LEMMA 2.2. Let $T: X \rightarrow Y$ and $S: Y \rightarrow Z$ be bounded linear operators between Banach spaces. Then the following are equivalent:

(1) $\operatorname{ker}(S \circ T)=\operatorname{ker}(T)$ and $\operatorname{Rng}(S \circ T)=\operatorname{Rng}(S)$.

(2) $Y=\operatorname{Rng}(T) \oplus \operatorname{ker}(S)$.

Furthermore in case (1) and (2) holds, then $\operatorname{Rng}(T)$ is closed in $Y$.

Proof. We first show (2) implies (1). Clearly $\operatorname{ker}(T) \subset \operatorname{ker}(S \circ T)$. So let $x \in \operatorname{ker}(S \circ T)$. Then $T(x) \in \operatorname{Rng}(T) \cap \operatorname{ker}(S)$. Thus from (2) we may conclude that $T(x)=0$ and so $x \in \operatorname{ker}(T)$.

It is also clear that $\operatorname{Rng}(S \circ T) \subset \operatorname{Rng}(S)$. To show $\operatorname{Rng}(S) \subset \operatorname{Rng}(S \circ T)$ let $z=S(y)$. Then using (2) we may write $y=T(x)+j$ where $(j)=0$. Hence $z=S(y)=S(T(x))$.

We now show (1) implies (2). Let $y \in Y$. By assumption there is an $x \in X$ such that $(S \circ T)(x)=S(y)$. We may write $y=T(x)+(y-T(x))$. Note $S(y-T(x))=0$ and so we may write $Y=\operatorname{Rng}(T)+\operatorname{ker}(S)$.

Now suppose $y \in \operatorname{Rng}(T) \cap \operatorname{ker}(S)$. Then $y=T(x)$ for some $x \in X$ and $S(y)=(S \circ T)(x)=0$. Thus $x \in \operatorname{ker}(S \circ T)$. But by assumption then $x \in$ $\operatorname{ker}(T)$ and so $y=T(x)=0$.

To complete the proof simply note $\operatorname{ker}(S)$ is closed in $Y$ and apply Lemma 2.1. Q.E.D.

It is interesting to note that condition (1) is sufficient along with the continuity of $S$ and $T$ to guarantee that $T$ has closed range. No further topological assumptions are required.

3. The Fredholm alternative for differential operators over compact manifolds. Let $M$ be a fixed compact $n$-manifold. Let $E$ and $F$ be two smooth vector bundles over $M$. Also assume each bundle has a Riemannian structure (i.e. a smoothly varying inner product on each fiber). A standard example of 
such a bundle is a tensor bundle over a Riemannian manifold $(M, g)$. Each tensor bundle has a Riemannian structure induced by $g$ (see [1]).

Without loss of generality we may assume $M$ has a given volume form $\mu$.

With this structure the Sobolev spaces of sections $W^{p, s}(E)$ and $W^{p, s}(F)$ may be defined for $s \in \mathbf{N}$ and $p \geqslant 1$. One approach is to construct a Riemannian connection for each bundle and to define for $f \in C^{\infty}(E)$ (for example),

$$
|f|_{p, s}=\sum_{|\alpha|<s}\left(\int_{M}\left\langle\nabla^{\alpha} f, \nabla^{\alpha} f\right\rangle_{E}^{p / 2}\right)^{1 / p}
$$

where $\langle,\rangle_{E}$ is the appropriate inner product induced by the metric structure on $E$. Then $W^{p, s}$ is the completion on $C^{\infty}(E)$ with respect to ||$_{p, s}$.

Another approach is to pick a finite trivializing cover for $E$. Then define the ||$_{p, s}$ norm in terms of these coordinate charts. This is independent of the choice of cover. See [25] for details.

As usual we define the topological dual of $W^{p, s}$ to be $W^{p^{\prime},-s}$ where $1 / p+1 / p^{\prime}=1$. The norm topology induces a natural norm on $W^{p^{\prime},-s}$. We will denote this as well by ||$_{p^{\prime},-s}$.

An important property of Sobolev spaces is the following.

Theorem 3.1 (Rellich-Kondrasov Compactness Theorem). Let $E$ be $a$ vector bundle with a smooth Finsler structure (i.e. a norm defined on each fiber) over a compact manifold $M$. Then if $p>1, s_{1}<s_{2}, W^{p, s_{1}}(E)$ is embedded compactly in $W^{p, s_{2}}(E)$.

For a proof see [25].

The theorem fails generally if $M$ is not compact.

Theorem 3.2 (Sobolev Embedding Theorem). Let $s>n / p+k$ then $W^{p, s}(E) \subset C^{k}(E)$ continuously.

The following theorems may also be found in [25].

THEOREM 3.3. Let " $\times$ " denote any bilinear map. Then

1. Pointwise application of $\times$ induces a continuous map from $W^{p, t_{1}} \times W^{p, t_{2}}$ to $L^{p}$ if $t_{1}+t_{2}>n / p$.

2. Let $s>n / p$ and $0 \leqslant t \leqslant s$ then pointwise application of $\times$ induces $a$ continuous map from $W^{p, s} \times W^{p, t} \rightarrow W^{p, t}$.

The above theorem works over $\mathbf{R}^{n}$ as well as compact manifolds. In fact a stronger version is proven in $\$ 5$.

There is the notion of a differential operator $D: C^{\infty}(E) \rightarrow C^{0}(F)$. (We do not require the coefficients to be $C^{\infty}$.) An invariant discussion of differential operators may be found in [25] or [26]. However it suffices to use trivializing covers for $E$ and $F$. In this way $D$ may locally be expressed as a differential operator from $C^{\infty}\left(U, \mathbf{R}^{m_{1}}\right)$ to $C^{0}\left(U, \mathbf{R}^{m_{2}}\right)$ where $m_{1}$ and $m_{2}$ are the dimensions of the fibers of $E$ and $F$, respectively, and $U$ is open in $\mathbf{R}^{n}$. The order of $D$ is a coordinate invariant. A linear operator of order $k$ has the local expression $D=\sum_{|\alpha| \leqslant k} a_{\alpha} D^{\alpha}$ where each coefficient $a_{\alpha}(p):\left(\mathbf{R}^{m_{1}}\right) \rightarrow \mathbf{R}^{m_{2}}$ is linear for each $p \in M$. 
If $D: C^{\infty}(E) \rightarrow C^{0}(F)$ is of order $k$ and has sufficiently smooth coefficients then by closure $D$ extends to an operator from $W^{p, s}(E)$ to $W^{p, s-k}(F)$. We may also define the formal adjoint $D^{*}: C^{\infty}(F) \rightarrow C^{0}(E)$ so that for all $e \in C^{\infty}(E)$ and $f \in C^{\infty}(F)$ we have

$$
\int_{M}\langle D e, f\rangle_{F} d \mu=\int_{M}\left\langle e, D^{*}(f)\right\rangle_{E} d \mu .
$$

Of course, $D^{*}$ also extends to $W^{p, s}(F)$ for each appropriate $p$ and $s$. The defining relation (4) holds whenever the integrals are defined.

For any differential operator $D$ there is a map $\sigma$ called the symbol of $D$ which associates to each cotangent vector $t \in T_{p}^{*}(M)$ linear map $\sigma_{t}: E_{p} \rightarrow F_{s}$. Also $\sigma_{t}\left(D^{*}\right)=\left(\sigma_{t}(D)\right)^{*}$, using the Riemannian structures on $E$ and $F$.

Definition 3.4. We say a differential operator $D$ has injective (resp. surjective) symbol if $\sigma_{t}(D)$ is injective (resp. surjective) for all nonzero $t . D$ is called elliptic if $\sigma_{t}(D)$ is an isomorphism for each nonzero $t$.

Note that if coordinates are used then the definitions of 'symbol' and 'elliptic' reduce to those usually given in partial differential equations texts.

The following is immediate from the definition:

PROPOSITION 3.5. If $D$ is a differential operator with injective symbol, then $D^{*} D$ is elliptic.

THEOREM 3.6 (A PRIORI ESTIMATE). Let $D$ be an elliptic operator of order $m$. Suppose locally $D=\sum_{|\alpha| \leqslant m} a_{\alpha} D^{\alpha}$ where each $a_{\alpha} \in W^{p, s_{|\alpha|}}$ with $s_{k}>n / p+k$ $-m+1$ and $s_{k}>s-m$ for some $s \in \mathbf{Z}$. Then there is $a C>0$ depending on $s, p$ and $D$ such that

$$
|f|_{p, s} \leqslant C\left(|D f|_{p, s-m}+|f|_{p}\right)
$$

for all $f \in L^{p}$ such that $D f \in W^{p, s-m}$.

This sort of estimate is standard for operators with smooth coefficients over regions in $\mathbf{R}^{n}[2, \mathbf{1 8}]$. Also the extension of this sort of estimate to operators with smooth coefficients over compact manifolds is found in [26]. The extension to operators with coefficients in $W^{p, s_{|\alpha|}}$ is due to Choquet-Bruhat and Christodoulou [13].

We will prove some important consequences of these elementary theorems.

THEOREM 3.7. Suppose $D$ is an elliptic operator whose coefficients satisfy the hypotheses of Theorem 3.6. Then if $f \in L^{p}$ and $D(f) \in L^{p}$ then $f \in W^{p, s}$.

COROLlaRY 1. The kernel of $D$ in $W^{p, t}$ is independent of $t$ for $t \leqslant s$.

Corollary 2. If $s>n / p+\mu$ then the kernel of $D$ in $L^{p}$ consists of $C^{\mu}$ functions.

THEOREM 3.8. Let $D$ be an elliptic operator satisfying the hypotheses of Theorem 3.6. Then the kernel of $D$ in $L^{p}$ is finite dimensional.

Proof. We cite a well-known result of functional analysis that a Banach space $B$ is finite dimensional if and only if the unit sphere in $B$ is compact. 
So let $f_{i}$ be a sequence in $\operatorname{ker}(D) \subset L^{p}$ such that $\left|f_{i}\right|_{p}=1$. Using Theorem 3.5 we may conclude that

$$
\begin{aligned}
\left|f_{i}\right|_{p, m} & \leqslant C(p, s+k)\left|f_{i}\right|_{p}, \\
& \leqslant C(p, s+k)\left|f_{i}\right|_{p} \leqslant C(p, s+k) .
\end{aligned}
$$

Thus $\left\{f_{i}\right\}$ is bounded in $W^{p, m}$. It follows from the Rellich-Kondrasov theorem that $f_{i}$ has a convergent subsequence in $L^{p}$. Thus every sequence in the unit sphere in $\operatorname{ker}(D)$ has a convergent subsequence. Q.E.D.

THEOREM 3.9. Let $p>1, s \geqslant m$ and $D$ be an elliptic operator of order $m$ whose coefficients satisfy the hypotheses of Theorem 3.6. Then $D\left(W^{p, s}\right)$ is closed in $W^{p, s-m}$.

Proof. We may write $W^{p, s}$ as $W^{p, s}=\operatorname{ker}(D) \oplus K$ where $K$ is closed in $W^{p, s}$. This is true since $\operatorname{ker}(D)$ is finite dimensional. Note $\alpha(k)=\alpha\left(W^{p, s}\right)$.

Claim. There is a constant $C$ such that $|f|_{p, s} \leqslant C|D(f)|_{p, s-m}$ for all $f \in K$.

If the claim does not hold there is a sequence $\left\{f_{i}\right\} \subset K$ such that $\left|f_{i}\right|_{p, s}=1$ and $D\left(f_{i}\right) \rightarrow 0$ in $W^{p, s-k}$. It follows from Theorem 3.1 that by passing to a subsequence we may assume $\left\{f_{i}\right\}$ is Cauchy in $L^{p}$. Clearly $\left\{D\left(f_{i}\right)\right\}$ is Cauchy. Thus from Theorem 3.6 we conclude $\left\{f_{i}\right\}$ is Cauchy in $W^{p, s}$. Hence $f_{i}$ converges to $\bar{f}$ in $K$. By continuity $D(\bar{f})=0$, and $|\bar{f}|_{p, s}=1$. However, this is impossible since $\operatorname{ker}(D) \cap K=\{0\}$.

To complete the proof let $g_{i}$ be a convergent (hence Cauchy) sequence in $D\left(W^{p, s}\right)$. Let $f_{i} \in K$ be such that $D\left(f_{i}\right)=g_{i}$. It follows from the claim that $f_{i}$ is Cauchy in $K$. Hence $f_{i}$ converges to $f$ in $K$. By continuity $D\left(f_{i}\right)$ converges to $D(\bar{f})=\lim \left(g_{i}\right)$. Hence the limit of $g_{i} \in D\left(W^{p, s}\right)$. Q.E.D.

Let us now recall an elementary result of functional analysis.

THEOREM 3.10. Let $T: X \rightarrow Y$ be a bounded linear transformation between reflexive Banach spaces. Suppose $T$ has closed range. Then for the adjoint of $T$, $T^{*}: Y^{*} \rightarrow X^{*}$ we have $\operatorname{ker}(T)^{\perp}=T^{*}\left(Y^{*}\right)$. Also the range of $T^{*}$ is closed and so $\operatorname{ker}\left(T^{*}\right)^{\perp}=T(X)$.

For a proof of this see [31].

An immediate consequence of the preceding is the following theorem.

THEOREM 3.11. Let $p>1, s>m$ and $D$ an elliptic operator satisfying the hypotheses of Theorem 3.6. Then

$$
D\left(W^{p, s}\right)=\left\{f \in W^{p, s-m}: \int\langle f h\rangle d \mu=0 \text { for all } h \in L^{p^{\prime}}, D^{*}(h)=0\right\} .
$$

Proof. We first check the case when $m=s$. It follows from 3.9 and 3.10 that $D\left(W^{p, m}\right)=\operatorname{ker}\left(D^{*}\right)^{\perp}$ since $\left(L^{p}\right)^{*}=L^{p^{\prime}}$ and the dual of $D$ is exactly $D^{*}$.

If $s>m$, then clearly for $f \in W^{p, s}, \int\langle D(f), h\rangle d \mu=0$ for $D^{*}(h)=0$, $h \in L^{p^{\prime}}$. Suppose $g \in W^{p, s-m}$ and $\int g h d \mu=0$ for all $h \in L^{p^{\prime}}$ such that $D^{*}(h)=0$. Then $g=D(\bar{f})$ for some $\bar{f} \in W^{p, m}$. However

$$
|\bar{f}|_{p, s} \leqslant C\left(|D \bar{f}|_{p, s-m}+|\bar{f}|_{p}\right) \leqslant C\left(|g|_{p, s-m}+|\bar{f}|_{p}\right) .
$$

Hence $g \in D\left(W^{p, s}\right)$. Q.E.D. 
It turns out that Theorem 3.11 is the one that generalizes in a natural way to the noncompact setting. However if the coefficients of $D$ are assumd to be sufficiently smooth then a more elegant theorem is possible.

THEOREM 3.12. Let $p>1, m$ an even positive number, $s>n / p+m$ and $D$ an elliptic operator satisfying the hypotheses of Theorem 3.6. Then if $m \leqslant t \leqslant s$ we have for $D: W^{p, t} \rightarrow W^{p, t-m}$,

$$
W^{p, t-m}=D\left(W^{p, t}\right) \oplus \operatorname{ker} D^{*} .
$$

Furthermore the range of $D$ is closed and the summands are $L^{2}$ orthogonal.

Proof. Using the local formula for $D^{*}$ (ignoring metric coefficients which are $C^{\infty}$ ), we find

$$
D^{*}(g)=\sum_{|\alpha|<m}(-1)^{|\alpha|} D^{\alpha}\left(d_{\alpha} g\right)=\sum_{|\alpha|<m}(-1)^{|\alpha|} \sum_{\beta<\alpha}\left(\begin{array}{c}
\beta \\
\alpha
\end{array}\right) D^{\alpha-\beta} a_{\alpha} D^{\beta} g .
$$

The coefficient of $D^{\beta} g$ belongs to $W^{p, t}$ where $t>n / p+|\alpha|-m+1-$ $(|\alpha|-|\beta|)=n / p+|\beta|-m+1$. Thus the hypotheses of Theorem 3.6 are satisfied for $D^{*}$ as an operator on $W^{p, s-m}$. Since $s-m>n / p h \in \operatorname{ker}\left(D^{*}\right) \subset$ $W^{p, s-m}$ then $f \mapsto \int_{M}\langle f, h\rangle d \mu$ is a bounded operator on $W^{p, t-m}$ (see Theorem 3.4). Since $\operatorname{ker}\left(D^{*}\right)$ is finite dimensional in $W^{p, s-m}$ (see Theorem 3.8) repeated application of the Hahn-Banach Theorem tells us that we may write

$$
W^{p, s-m}=\operatorname{ker}\left(D^{*}\right) \oplus\left\{f \in W^{p, s-m}: \int_{M}\langle f h\rangle=0 \text { for all } h \in \operatorname{ker}\left(D^{*}\right)\right\} .
$$

Using the previous theorem and part 1 (above) we see the second summand is $D\left(W^{p, s}\right)$. Q.E.D.

COROLlary. If $g \in W^{p, t-m}$, then $D(f)=g$ has a solution $f \in W^{p, t}$ iff

$$
\int_{M}\langle h, g\rangle d \mu=0 \text { for all } f \in \operatorname{ker}\left(D^{*}\right) \subset W^{p, s-m} .
$$

THEOREM 3.13. Let $\alpha: C^{\infty}(E) \rightarrow C^{0}(F)$ be an operator of order $k$ with injective (resp. surjective) symbol. Suppose the elliptic operator $\alpha^{*} \alpha$ (resp. $\alpha \alpha^{*}$ ) satisfies the hypotheses of Theorem 3.12 for $p>1$ and $s>n / p+2 k$. Then for $2 k \leqslant t \leqslant s$ (and if $\alpha$ has surjective symbol $s-k>t>n(1 / p-1 / 2)+k$ ), we have

$$
W^{p, t-k}(F)=\alpha\left(W^{p, t}\right) \oplus \operatorname{ker}\left(\alpha^{*}\right) .
$$

Furthermore, the range of $\alpha$ is closed in $W^{p, t-k}$.

Proof. We first consider the case where the symbol of $\alpha$ is injective.

Using Lemma 2.2 we need only show that $\operatorname{ker}\left(\alpha^{*} \alpha\right)=\operatorname{ker}(\alpha)$ and $\operatorname{Rng}\left(\alpha^{*} \alpha\right)=\operatorname{Rng}(\alpha)$ for the chain

$$
W^{p, t} \stackrel{\alpha}{\rightarrow} W^{p, t-k} \stackrel{\alpha^{*}}{\rightarrow} W^{p, t-2 k} .
$$


Let us assume $f \in \operatorname{ker}\left(\alpha^{*} \alpha\right)$. Then using Theorem 3.7 we have $\alpha(f) \in C^{k}$. Thus we have

$$
0=\int_{M}\left\langle f, \alpha^{*} \alpha f\right\rangle_{E} d \mu=\int_{M}\langle\alpha f, \alpha f\rangle_{F} d \mu .
$$

Hence $\alpha(f)=0$. Thus $\operatorname{ker}\left(\alpha^{*} \alpha\right) \subset \operatorname{ker}(\alpha)$. Clearly $\operatorname{ker}(\alpha) \subset \operatorname{ker}\left(\alpha^{*} \alpha\right)$.

Also, it is clear that $\operatorname{Rng}\left(\alpha^{*} \alpha\right) \subset \operatorname{Rng}\left(\alpha^{*}\right)$. So assume $f=\alpha^{*}(g)$ for $g \in$ $W^{p, t-k}$. Using Theorem 3.1 we may write $f=\alpha^{*} \alpha(h)+j$ where $\alpha^{*} \alpha(j)=0$. Then $j \in W^{p, s}$ and from the above reasoning $\alpha(j)=0$. Hence

$$
\begin{aligned}
\int_{M}\langle j, j\rangle d \mu & =\int\left\langle j, f-\alpha^{*} \alpha(h)\right\rangle d \mu=\int\left\langle j, \alpha^{*}(g)\right\rangle-\int\left\langle j, \alpha^{*} \alpha h\right\rangle d \mu \\
& =\int\langle\alpha(j), g\rangle d \mu-\int\left\langle\alpha^{*} \alpha(j), h\right\rangle d \mu=0-0=0 .
\end{aligned}
$$

Now suppose $\alpha$ has surjective symbol. In this case $\alpha \alpha^{*}$ is elliptic. Again we only need verify that $\operatorname{ker}\left(\alpha^{*} \alpha\right)=\operatorname{ker}(\alpha)$ and $\operatorname{Rng}\left(\alpha^{*} \alpha\right)=\operatorname{Rng}\left(\alpha^{*}\right)$. Since $\alpha^{*} \alpha$ is not elliptic we may not apply the regularity estimate. However since $t-k>n(1 / p-1 / 2)$ we know $W^{p, t-k} \subset L^{2}$ (see Palais [25]) and so the integration by parts argument used above to show $\operatorname{ker}\left(\alpha^{*} \alpha\right)=\operatorname{ker}(\alpha)$ is valid.

Now suppose $w \in W^{p, t-2 k}$ and $w=\alpha^{*}(v)$ where $v \in W^{p, t-k}$. Since $t-k$ $<s-2 k$ we may apply Theorem 3.12 to where $V=\alpha \alpha^{*}(u)+h$ where $u \in W^{p, t+k}$ and $\alpha \alpha^{*}(h)=0$. Then $w=\alpha^{*} \alpha\left(\alpha^{*} h\right)+\alpha^{*}(h)$. Hence we are finished if $\alpha^{*}(h)=0$. However we may apply the integration by parts argument again (in fact $\alpha^{*}(h)$ is continuous) to show

$$
0=\int\left\langle\alpha \alpha^{*}(h), h\right\rangle d \mu=\int\left\langle\alpha^{*}(h), \alpha^{*}(h)\right\rangle d \mu .
$$

Q.E.D.

It is interesting to note that Theorem 3.12 is really a special case of Theorem 3.13. Elliptic operators have injective symbol and surjective symbol. However Theorem 3.12 does seem to require separate proof.

There are some differences between operators with injective symbol and elliptic operators. An example is found in the following theorem.

Theorem 3.14 (Bourguignon-Ebin-MARSDEN). Let $\alpha$ be a differential operator with symbol that is injective but not surjective. Then $\operatorname{ker}\left(\alpha^{*}\right) \cap W^{p, s}$ is infinite dimensional. Also the cokernel is infinite dimensional.

A proof of this may be found in [5].

Thus in the elliptic case, $\operatorname{ker}\left(\alpha^{*}\right)$ is infinite dimensional.

4. Decomposition of tensor fields over compact manifolds. In this section we present some straightforward applications of the previous results. We will restrict ourselves to tensor fields defined over compact manifolds. Some discussion of the noncompact case will be given in the later sections.

Theorem 4.1 (Helmholtz Decomposition). Let $p>1, s>n / p+2$ and $g$ a Riemannian metric of class $W^{p, s}$. Then for $1 \leqslant t \leqslant s$

$$
W^{p, t-1}(T M)=\operatorname{grad}\left(W^{p, t}\right) \oplus \operatorname{ker}(\operatorname{div}) .
$$


Proof. This follows immediately from Theorem 3.13, noting that the symbol of grad is injective and the grad* = div, and the coefficients of grad are coordinates of the metric. Q.E.D.

REMARK. In the case $p=2$ and the metric is $C^{\infty}$ a proof of this is possible without the use of the Fredholm alternative.

The above result may be extended to the more general Hodge decomposition. Let $\Lambda^{k}$ be the bundle of alternating $k$-forms. The exterior derivative is a first order differential operator $d: C^{\infty}\left(\Lambda^{k}\right) \rightarrow C^{\infty}\left(\Lambda^{k+1}\right)$. If $\alpha \in L^{p}\left(\Lambda^{k}\right)$ and $\beta \in L^{p^{\prime}}\left(\Lambda^{k}\right), 1 / p+1 / p^{\prime}=1$, we may define $(\alpha, \beta)=\int_{M} \alpha \Lambda * \beta d \mu$ where $\Lambda$ is the wedge product and $*$ is the Hodge-star operator (see [28]). In this way we may identify $L^{p^{\prime}}\left(\Lambda^{k}\right)$ with the dual of $L^{p}\left(\Lambda^{k}\right)$. In this case we prove Theorem 4.1 directly from the fundamental lemma and the regularity theorem. First let $s=1$; using $\operatorname{div}_{g}: H^{1} \rightarrow L^{2}$ and $\operatorname{grad}_{g}: H^{2} \rightarrow H^{1}$, we need show (1) $\operatorname{ker}\left(\Delta_{g}\right)=\operatorname{ker}\left(\operatorname{grad}_{g}\right)$ and (2) $\Delta_{g}\left(H^{2}\right)=\operatorname{div}_{g}\left(H^{1}\right)$. Condition (1) follows immediately from the regularity theorem and integration by parts (or alternately the maximum principle). We get that $\operatorname{ker}\left(\Delta_{g}\right)=\operatorname{ker}\left(\operatorname{grad}_{g}\right)=\{$ constant functions . To show (2), note if $f=\operatorname{div}_{g}(u) \in L^{2}$ then the total integral of $f$ vanishes by the divergence theorem. Thus $f$ is orthogonal to the constants which form the null space of $\Delta_{g}^{*}\left(=\Delta_{g}\right)$ in $L^{2}$. Thus using a standard result of Hilbert space theory $f=\Delta_{g} u$. The case $s>1$ follows using regularity.

With this identification we have the codifferential $\delta: C^{\infty}\left(\Lambda^{k}\right) \rightarrow C^{\infty}\left(\Lambda^{k-1}\right)$ defined so that $\delta=d^{*}$. Thus $(d \alpha, \beta)=(\alpha, \delta \beta)$. Since $d^{2}=0$, we have $\delta^{2}=0$. The Laplace-Beltrami operator is defined as $\Delta=d \delta+\delta d$. Also note $d \Delta=\Delta d$ and $\delta \Delta=\Delta \delta$.

Theorem 4.2 (Hodge Decomposition). Let $p>1$ and $s \geq 0$. Then

$$
W^{p, s}\left(\Lambda^{k}\right)=d\left(W^{p, s+1}\left(\Lambda^{k+1}\right)\right) \oplus \delta\left(W^{p, s+1}\left(\Lambda^{k-1}\right)\right) \oplus \operatorname{ker}(\Delta)
$$

where the summands are closed, and $\operatorname{ker}(\Delta)$ is finite dimensional.

Proof. First recall some standard facts about $\Delta$. It is elliptic, selfadjoint, and has smooth coefficients. Also if $\Delta f=0$ then since $f \in C^{\infty}$ we find

$$
(d f, d f)=(\delta d f, f)=-(d \delta f, f)=-(\delta f, \delta f) .
$$

Thus both $(d f, d f)=0$ and $(\delta f, \delta f)=0$. In fact $d f=\delta f=0$.

It follows from Theorem 3.11 that $W^{p, s}\left(\Lambda^{k}\right)=\Delta\left(W^{p, s+2}\left(\Lambda^{k}\right)\right) \oplus \operatorname{ker}(\Delta)$. Clearly, if $f \in \Delta\left(W^{p, s+2}\left(\Lambda^{k}\right)\right)$ then $f$ may be written as $f=d \alpha+\delta \beta$ and so we may write

$$
W^{p, s}\left(\Lambda^{k}\right)=d\left(W^{p, s+1}\left(\Lambda^{k-1}\right)\right)+\delta\left(W^{p, s+1}\left(\Lambda^{k+1}\right)\right)+\operatorname{ker}(\Delta) .
$$

We need to show the sum is direct. Suppose $f=d \alpha+\delta \beta$ and $\Delta f=0$. Then since $d f=\delta f=0$ we have $\delta d \alpha=0$ and $d \delta \beta=0$. Also, using $(d \alpha, \delta \beta)=0$ we have

$$
(f, f)=(d \alpha, d \alpha)+(\delta \beta, \delta \beta)=(\alpha, \delta d \beta)+(\beta, d \delta \beta)=0
$$

and so $f=0$. Now if $f=d \alpha=\delta \beta$ then $d f=0$ and $\delta f=0$. Thus $\Delta f=0$ and the decomposition is established. 
It remains to show the summands are closed. To this end note that $\operatorname{ker}(d)=d\left(W^{p, s+1}\left(\Lambda^{k-1}\right)\right) \oplus \operatorname{ker}(\Delta)$. For suppose $f=d g+j$ where $\Delta j=0$. Then $d f=d j$. Now $d \Delta j=d \delta d j=0$. Thus if $j$ is $C^{\infty}$ we may calculate that

$$
0=(d \delta d j, d j)=(\delta d j, \delta d j)
$$

and so $\delta d j=0$. It follows $0=(\delta d j, j)=(d j, d j)$. Thus $d j=0$ and we have $d f=d j=0$. This shows $d\left(W^{p, s+1}\left(\Lambda^{k-1}\right)\right) \oplus \operatorname{ker}(\Delta) \subset \operatorname{ker}(d)$.

Now let $f \in \operatorname{ker}(d)$. We know $f=d \alpha_{1}+\delta \alpha_{2}+h$ where $\Delta(h)=0$. We need only that $\delta \alpha_{2}=0$. We have $d f=d \delta \alpha_{2}=0$ and so

$$
0=\left(d \delta \alpha_{2}, \alpha_{2}\right)=\left(\delta \alpha_{2}, \delta \alpha_{2}\right)
$$

and hence $\delta \alpha_{2}=0$.

Since $\operatorname{ker}(d)$ is closed and so is a Banach space and $\operatorname{ker}(\Delta)$ is closed in $\operatorname{ker}(d)$ then an application of Lemma 2.1 tells us the range of $d$ is closed.

The fact that the range of $\delta$ is closed follows immediately from one more application of Lemma 2.1. Q.E.D.

EXAMPLE. The canonical decomposition of symmetric 2-tensors (Berger and Ebin [4]).

Let $(M, g)$ be a compact Riemannian manifold. Let $S^{2}$ be the bundle of symmetric two tensors. Then for $X \in W^{p, s}(T M)$ let $K(X)=L_{X} g$ where $X$ is the Lie derivative. This is a first order differential symbol. In coordinates $K_{g}(X)_{i j}=X_{i \mid j}+X_{j \mid i}$. Thus the symbol $\sigma_{\xi}(K): T_{p} M \rightarrow S_{p}^{2}$ is given by $\left(V^{1}, \ldots, V^{n}\right) \rightarrow\left(\xi_{i} V^{j}+\xi_{j} V^{i}\right)$. Now setting $i=j$ then $\xi_{i} V^{i}=0$ (no summation) for all choices of $V^{i}$. Thus if $\xi_{j} \neq 0$ we must have $V^{j}=0$. Also then $\xi_{j} V^{i}=0$ for $i=1, \ldots, n$; thus $\left(V^{1}, \ldots, V^{n}\right)=0$ and the symbol is injective.

The formal adjoint of $K$ is computed by

$$
\int\left\langle K_{g}(X), S\right\rangle d \mu=-\int \alpha X \operatorname{div}_{g}(S) d \mu
$$

where $\operatorname{div}_{g}(S)^{i}=S_{\mid j}^{i j}$. Then we have the following theorem.

THEOREM 4.3. Let $p>1, s>n / p+2$ and $g$ a metric of class $W^{p, s}$. Then we have the following decomposition:

$$
W^{p, s}\left(S^{2}\right)=K_{g}\left(W^{p, s+1}(T M)\right) \oplus \operatorname{ker}\left(\operatorname{div}_{g}\right)
$$

where the range of $K_{g}$ is closed.

Proof. This is an immediate application of Theorem 3.12. One need only do a coordinate calculation to check the required smoothness of the coefficients of $K_{g}$. Q.E.D.

This example is a special case of a general class of decompositions that arise in theoretical mechanics [3].

We give two more applications of Theorem 3.13.

Proposition 4.4. Let $(M, g)$ be a $C^{\infty}$ Riemannian manifold and $f \in$ $C^{\infty}(M, \mathbf{R})$; then there is a $\chi \in C^{\infty}(T M)$ such that $\operatorname{div}_{g} \chi=f$ iff $\int_{M} f d \mu_{g}=0$. 
Proof. Let $p>1$. Then $f \in W^{p, s}$ for any $s$. We may write (since $\operatorname{div}_{g}$ has surjective symbol)

$$
W^{p, s}=\operatorname{div}_{g}\left(W^{p, s+1}\right) \oplus \operatorname{ker}\left(\operatorname{grad}_{g}\right) .
$$

Thus $f$ is a member of the range of $\operatorname{div}_{g}$ iff $f$ is $L^{2}$ orthogonal to the kernel of $\operatorname{grad}_{g}$, i.e. the constants. Thus if $\int f d \mu_{g}=0$ we may write $f=\operatorname{div}_{g} \chi$ where $\chi \in W^{p, s+1}$ for any s. Hence $\chi \in C^{\infty}$. Q.E.D.

Proposition 4.5. Let $(M, g)$ be a $C^{\infty}$ Riemannian manifold. Let $\chi \in$ $C^{\infty}(T M)$. Then $\chi=\operatorname{div}_{g}(S)$ where $S$ is a bilinear symmetric 2-tensor iff $\int_{M}\langle X, h\rangle_{g} d \mu$ for all killing fields $h$ on $M$ (i.e. $h \in \operatorname{ker}\left(K_{g}\right)$ ).

Proof. This is proven similarly as the previous Proposition 4.4 using the decomposition

$$
W^{p, s}(T M)=\operatorname{div}_{g}\left(S^{p, s+1}\right) \oplus \operatorname{ker}_{g}\left(K_{g}\right) \text {. Q.E.D. }
$$

5. Weighted Sobolev spaces over $\mathbf{R}^{n}$. Research over the last few years has shown that an appropriate setting for studying homogeneous elliptic operators on $\mathbf{R}^{n}$ are the weighted Sobolev spaces $M_{s, \delta}^{p}$ defined below. These spaces were suggested by inequalities found in a paper by Nirenberg and Walker [24]. They were first defined and applied by the author [6-12]. More recently R. McOwen [23], R. Lockhardt [20] and Christodoulou and Choquet-Bruhat [13] have extended the previous known results and helped clarify the previous work. Also, the use of $M_{s, \delta}^{p}$ spaces for the study of hyperbolic operators has begun (see [14]).

Definition 5.1. Let $s \in \mathbf{N}, 1 \leqslant p \leqslant \infty$ and $\delta \in \mathbf{R}$, and $\sigma(x)=(1+$ $\left.|x|^{2}\right)^{1 / 2}$

$$
|f|_{p, s, \delta}=\sum_{|\alpha|<s}\left|\sigma^{\delta+|\alpha|} D^{\alpha} f\right|_{p}
$$

where ||$_{p}$ is the standard $L^{p}$ norm on $\mathbf{R}^{n}$.

The completion of $C_{0}^{\infty}\left(\mathbf{R}^{n}, \mathbf{R}^{m}\right)$ with respect to $\left.\right|_{p, s, \delta}$ is called $M_{s, \delta}^{p}\left(\mathbf{R}^{n}, \mathbf{R}^{m}\right)$.

As is customary we will denote $M_{s, \delta}^{p}\left(\mathbf{R}^{n}, \mathbf{R}^{m}\right)$ by $M_{s, \delta}^{p}$ when there is no danger of confusion.

In order to get a feeling for some of the technical results to follow note that $\sigma^{\alpha}(x)=\left(1+x^{2}\right)^{\alpha / 2}$ belongs to $M_{s, \delta}^{p}$ if and only if $\alpha<-(n / p+\delta)$. Thus, for example, if $-n / p<\delta<-n / p+1$ then $M_{s, \delta}^{p}$ includes $\sigma^{-1}$ but not $\sigma^{0}=1$. Also we may include linear functions in $M_{s, \delta}^{p}$ by letting $\delta<-n / p-1$, etc.

The next theorem (following a series of useful lemmas) shows that if $s>n / p$ the above reasoning has a partial converse. That is, if $f \in M_{s, \delta}^{p}$ for $s>n / p$, then at infinity $f=o\left(\sigma^{\alpha}\right)$ for $\alpha<-(n / p+\delta)$.

LEMMA 5.1. For $\delta \in \mathbf{R}$ and $\alpha$ a multi-index we have

$$
\begin{aligned}
& 0<\lim _{|x| \rightarrow \infty} \frac{\left|D^{\alpha} \sigma^{\delta}(x)\right|}{|x|^{\delta-|\alpha|}}<\infty, \\
& 0<\lim _{|x| \rightarrow \infty} \frac{\left|D^{\alpha} \sigma^{\delta}(x)\right|}{\sigma^{\delta-|\alpha|}(x)}<\infty .
\end{aligned}
$$


This is proven by direct calculation.

LeMmA 5.2. For any $p \geqslant 1, s \in \mathbf{N}$ and $\delta, l \in \mathbf{R}$ the map $f \mapsto f \sigma^{l}$ is a continuous map from $M_{s, \delta}^{p}$ into $M_{s, \delta-l}^{p}$.

Proof. We will show in general that there is a $C>0$ such that

$$
\left|f \sigma^{l}\right|_{p, s, \delta-l} \leqslant C|f|_{p, s, \delta} .
$$

It suffices to show if $\beta$ is a multi-index and $|\beta|=s$, then

$$
\left|D^{\beta}\left(f \cdot \sigma^{l}\right) \sigma^{\delta-l+s}\right|_{p} \leqslant C|f|_{p, s, \delta}
$$

To show this we write

$$
D^{\beta}\left(f \cdot \sigma^{l}\right)=\sum_{\gamma<\beta}\left(\begin{array}{l}
\gamma \\
\beta
\end{array}\right) D^{\gamma} f D^{\beta-\gamma} \sigma^{l} .
$$

Thus using Lemma 5.1 we find

$$
\begin{aligned}
\left|D^{\beta}\left(f \cdot \sigma^{l}\right) \sigma^{\delta-l+s}\right|_{p} & \leqslant C \sum_{\gamma<\beta}\left|D^{\gamma} f \sigma^{l-s+|\gamma|} \sigma^{\delta-l+s}\right|_{p} \\
& \leqslant C \sum_{|\gamma|<s}\left|D^{\gamma} f \sigma^{\delta+|\gamma|}\right|_{p} \leqslant C|f|_{p, s, \delta} \text {. Q.E.D. }
\end{aligned}
$$

The next lemma is a Sobolev type inequality.

LeMmA 5.3. Let $s>n / p$ and $\delta<n / p$. There is a constant $C>0$ such that for all $f \in C_{0}^{\infty}$

$$
\left\|f \sigma^{\delta}\right\| \leqslant C|f|_{p, s, 0}
$$

Proof. Let $f \in C_{0}^{\infty}$. Then for any $x_{0} \in \mathbf{R}^{n}$ and $\theta \in S^{n-1}=\left\{y \in \mathbf{R}^{n}\right.$ : $|y|=1\}$ we have

$$
f\left(x_{0}\right) \sigma^{\delta}\left(x_{0}\right)=-\int_{0}^{\infty} \frac{d}{d t}\left(f \cdot \sigma^{\delta}\left(x_{0}+t \theta\right)\right) d t .
$$

Integrate by parts $(s-1)$ times to get

$$
\begin{aligned}
f\left(x_{0}\right) \sigma^{\delta}\left(x_{0}\right) & =\frac{(-1)^{s}}{(s-1) !} \int_{0}^{\infty} \frac{d^{s}}{d t^{s}}\left(f \cdot \sigma^{\delta}(x+t \theta)\right) t^{s-1} d t \\
& =\frac{(-1)^{s}}{(s-1) !} \int_{0}^{\infty} \sum_{l=0}^{s}\left(\begin{array}{l}
l \\
s
\end{array}\right) \frac{d^{l} f}{d t^{l}}\left(x_{0}+t \theta\right) \frac{d^{s-\sigma^{\delta}}}{d t^{s-l}}\left(x_{0}+t \theta\right) t^{s-1} d t
\end{aligned}
$$

Thus

$$
\left|f\left(x_{0}\right) \sigma^{\delta}\left(x_{0}\right)\right| \leqslant C_{1} \int_{0}^{\infty} \sum_{l=0}^{s}\left|\frac{d^{l} f\left(x_{0}+t \theta\right)}{d t^{l}}\right|\left|\frac{d^{s-l} \sigma^{\delta}}{d t^{s-l}}\left(x_{0}+t \theta\right)\right| t^{s-1} d t .
$$


Now integrate both sides over $S^{n-1}$ to get

$$
\begin{gathered}
w_{n-1}\left|f\left(x_{0}\right) \sigma^{\delta}\left(x_{0}\right)\right| \\
\leqslant C_{1} \int_{S^{n-1}} \int_{0}^{\infty} \sum_{l=0}^{s}\left|\frac{d^{l} f}{d t^{l}}\left(x_{0}+t \theta\right)\right|\left|\frac{d^{s-l} \sigma^{\delta}}{d t^{s-l}}\left(x_{0}+t \theta\right)\right| t^{s-n} t^{n-1} d t d S
\end{gathered}
$$

where $w_{n-1}$ is the $(n-1)$-volume of $S^{n-1}$. After dividing by $w_{n-1}$ and applying Lemma 5.1 we conclude

$$
\left|f\left(x_{0}\right) \sigma^{\delta}\left(x_{0}\right)\right| \leqslant C_{2} \int_{\mathbf{R}^{n}} \sum_{l=0}^{s}\left|D^{l} f(y)\right|\left|\sigma^{\delta+l-s}(y)\right|\left|y-x_{0}\right|^{s-n} d V(y)
$$

where $D^{l} f(y)$ is the total $l$ th derivative. Thus using Hölder's inequality if $1 / p+1 / p^{\prime}=1$ we have

$$
\begin{aligned}
\left|f\left(x_{0}\right) \sigma^{\delta}\left(x_{0}\right)\right| \leqslant & C_{2} \sum_{l=0}^{s}\left(\int_{\mathbf{R}^{n}}\left|D^{l} f \sigma^{l}\right|^{p} d V\right)^{1 / p} \\
& \cdot\left(\int_{\mathbf{R}^{n}}\left(\left|\sigma^{\delta-s}(y)\right|\left|y-x_{0}\right|^{s-n}\right)^{p^{\prime}} d V\right)^{1 / p^{\prime}} .
\end{aligned}
$$

Thus we need to check that the second integral in each summand converges. This requires first that the singularity at $x_{0}$ is well behaved. This requires

$$
(s-n) p / p-1+n>0 .
$$

This is equivalent to $s>n / p$.

Second, we must check the growth at infinity. By checking limits at infinity we may treat $\sigma$ as $|y|,\left|x_{0}-y\right|$ as $|y|$, and $\sigma^{\delta-s}(y)$ as $|y|^{\delta-s}$. Thus the integral converges at infinity iff

$$
((\delta-s)+(s-n)) p^{\prime}+n<0 .
$$

That is,

$$
(\delta-n) p /(p-1)+n<0 .
$$

This is equivalent to $\delta<n / p$. Thus $\left|f\left(x_{0}\right) \sigma^{\delta}\left(x_{0}\right)\right| \leqslant C|f|_{p, s, 0}$. Since this holds for all $x_{0} \in \mathbf{R}^{n}$ the lemma is proved. Q.E.D.

TheOREM 5.4. Let $p>1, s>n / p$ and $\delta \in \mathbf{R}$. Then if $f \in M_{s, \delta}^{p}$ and $\beta<$ $n / p+\delta$ we have $\lim _{|x| \rightarrow \infty}\left|f(x) \sigma^{\beta}(x)\right|=0$.

Proof. Let $\theta_{R}$ be a $C^{\infty}$ function satisfying

$$
\theta_{R}= \begin{cases}0, & |x| \leqslant R, \\ 1, & |x| \geqslant 2 R .\end{cases}
$$

Also we may assume $\left|D^{\alpha} \theta_{R}(x)\right|<C_{\alpha} R^{-\alpha}$ for some $C_{\alpha}$.

Claim. If $g \in M_{s, 0}^{p}$ then $\lim _{R \rightarrow \infty}\left|\theta_{R} g\right|_{p, s, 0}=0$. To see this note that by using total derivatives for $\alpha \leqslant s$

$$
\left|D^{\alpha}\left(\theta_{R} g\right) \sigma^{\alpha}\right|_{p} \leqslant C \sum_{l=0}^{\alpha}\left|D^{l} g\left(D^{\alpha-l} \theta_{R}\right) \sigma^{\alpha}\right|_{p}
$$

There are two cases to consider. 
Case (1). $l=\alpha$. In this case we check for each $x$,

$$
\left|D^{\alpha} g(x) \theta_{R}(x) \sigma^{\alpha}(x)\right|^{p} \leqslant\left|D^{\alpha} g \sigma^{\alpha}(x)\right|^{p}
$$

and so by the dominated convergence theorem $\lim _{R \rightarrow \infty}\left|D^{\alpha} g \theta_{R}(x) \sigma^{\alpha}(x)\right|_{p}=0$.

Case (2). $l<\alpha$. In this case the support of $D^{\alpha-l} \theta_{R}$ is contained in $\{R \leqslant|x| \leqslant 2 R\}$ and so using the estimate on $\left\|D^{\alpha-1} \theta_{R}\right\|$ and Lemma 5.1 we have

$$
\left\|\left(D^{\alpha-l} \theta_{R}\right) \sigma^{\alpha-l}\right\| \leqslant C R^{l-\alpha}(2 R)^{\alpha-l} .
$$

Hence there is a constant $C$ such that

$$
\begin{aligned}
\left|D^{l} g(x) D^{\alpha-l} \theta_{R}(x) \sigma^{\alpha}(x)\right|^{p} & \leqslant\left\|D^{\alpha-l} \theta_{R} \sigma^{\alpha-l}\right\|^{p}\left|D^{l} g(x) \sigma^{l}(x)\right|^{p} \\
& \leqslant C\left|D^{l} g(x) \sigma^{l}(x)\right|^{p}
\end{aligned}
$$

Thus we may again use the dominated convergence theorem to conclude $\lim _{R \rightarrow \infty}\left|D^{l} g D^{\alpha-l} \theta_{R} \sigma^{\alpha}\right|_{p}=0$.

The claim now follows.

Now set $g=f_{\sigma}{ }^{\delta}$. Then from Lemma $5.2 g \in M_{s, 0}^{p}$. Now we have, using Lemma 5.3, for all $\gamma<n / p$ and $R>0$,

$$
\lim _{|x| \rightarrow \infty}\left|g(x) \sigma^{\gamma}(x)\right|=\lim _{|x| \rightarrow \infty}\left|\theta_{R} g(x) \sigma^{\gamma}(x)\right| \leqslant \lim _{|x| \rightarrow \infty} C\left|\theta_{R} g\right|_{p, s, 0} .
$$

Note $\left|\theta_{\mathrm{R}} \mathrm{g}\right|_{p, s, 0}$ is independent of $|x|$ and so we have for all $R$

$$
0 \leqslant \lim _{|x| \rightarrow \infty}\left|g(x) \sigma^{\gamma}(x)\right| \leqslant C\left|\theta_{R} g\right|_{p, s, 0}
$$

Using the claim and letting $R \rightarrow \infty$ we find $\lim _{|x| \rightarrow \infty}\left|g(x) \sigma^{\gamma}(x)\right|=0$. Thus $\lim _{|x| \rightarrow \infty}\left|f(x) \sigma^{\delta+\gamma}(x)\right|=0$ and set $\beta=\delta+\gamma$ to finish the proof. Q.E.D.

Let us give some examples of applications of the previous theorem.

EXAMPLE 1. Let $n=3$ and $p=2$ and $s>3 / 2$. Then for $f \in M_{s, \delta}^{2}$ we know $\lim _{|x| \rightarrow \infty}|f(x)|=0$ if $\delta>-3 / 2$.

EXAMPLE 2. Let $p>1$ and $f \in M_{s, 0}^{p}$ for $s>n / p$. Then $\lim _{|x| \rightarrow \infty}\left|f(x) \sigma^{\delta}(x)\right|$ $=0$ for $\delta<n / p$. If we wish that $f(x)$ "fall off like" $\sigma^{2-n}$ at infinity (this is important for the study of second-order operators) then we wish $\lim _{|x| \rightarrow \infty}\left|f(x) \sigma^{\delta}(x)\right|=0$ for all $\delta<n-2$. This requires $n / p<n-2$ or equivalently $p>n / n-2$. Thus $f \in M_{s, 0}^{p}$ falls like $\sigma^{2-n}$ if $s>n / p$ and $p>n / n-2$.

If $s_{1}>n / p$ and $s_{2} \leqslant s_{1}$ it is easy to show that pointwise multiplication induces a continuous map from $M_{s_{1}, \delta_{1}}^{p} \times M_{s_{2}, \delta_{2}}^{p}$ into $M_{s_{2}, \delta_{1}+\delta_{2}}^{p}$. However one can in fact do better. Consider the case where $\delta_{1}=\delta_{2}=0$ and $s_{1}=s_{2}$. In the presence of this much smoothness one might expect that for $f \in M_{s, 0}^{p}, f^{2}$ falls off faster than $f$. Also if $\delta>-n / p$ then if $f \in M_{s, \delta}^{p}, f$ approaches zero at infinity (Theorem 5.4). Thus one might expect that $M_{s, \delta}^{p}$ is closed under multiplication if $s>n / p$ and $\delta>-n / p$. Both of these expectations are justified as is shown below. This phenomenon (for $p=2$ ) was first established by Choquet-Bruhat and Christodoulou [13]. The proofs given here were found independently by the author. 
LEMMA 5.5. Let $p>1, s_{1}, s_{2}$ be such that $s_{1}+s_{2}>n / p$. Then pointwise multiplication induces a continuous map from $M_{s_{1}, \delta_{1}}^{p} \times M_{s_{2}, \delta_{2}}^{p} \rightarrow M_{0, \delta_{1}+\delta_{2}+\alpha}^{p}$ where $\alpha<n / p$.

Proof. Using Lemma 5.2, it is sufficient to show this theorem when $\delta_{1}=\delta_{2}=0$. Let

$$
\begin{aligned}
p_{1} & =\left(s_{1}+s_{2}\right) p / s_{2}, & p_{2} & =\left(s_{1}+s_{2}\right) p / s_{1}, \\
t_{1} & =s_{1} \alpha /\left(s_{1}+s_{2}\right), & t_{2} & =s_{2} \alpha /\left(s_{1}+s_{2}\right) .
\end{aligned}
$$

Note $1 / p_{1}+1 / p_{2}=1 / p$ and $t_{1}+t_{2}=\alpha$. Then for $f_{i} \in M_{s_{i}, 0}^{p}, i=1,2$, we have

$$
\left|f_{1} \cdot f_{2} \sigma^{\alpha}\right|_{p} \leqslant\left|f_{1} \sigma^{\alpha^{t_{1}}}\right|_{p_{1}}\left|f_{2} \sigma^{\alpha^{t_{2}}}\right|_{p_{2}} .
$$

Now for $i=1,2$ we have

$$
\left|f_{i} \sigma^{t_{i}}\right|_{p_{i}} \leqslant C\left|D^{s_{i}}\left(f_{i} \sigma^{t_{i}}\right)\right|_{q_{i}}
$$

where $1 / p_{i}=1 / q_{i}-s_{i} / n$ (see [25]).

Repeated use of the product rule and the fact that $\left|D^{\beta} \sigma^{l}\right| \leqslant \mathrm{Co}^{l-\beta}$ where $C$ depends on $\beta$ and $l$ yields

$$
\begin{aligned}
\left|f_{i} \sigma^{t_{i}}\right|_{p_{i}} & \leqslant C \sum_{\gamma=0}^{s_{i}}\left|D^{\gamma} f_{i} \sigma^{t_{i}-s_{i}+\gamma}\right|_{q_{i}} \leqslant C \sum_{\gamma=0}^{s_{i}}\left|\left(D^{\gamma} f_{i} \sigma^{\gamma}\right) \sigma^{t_{i}-s_{i}}\right|_{q_{i}} \\
& \leqslant C \sum_{\gamma=0}^{s_{i}}\left|D^{\gamma} f_{\sigma^{\gamma}}\right|_{p}\left|\sigma^{t_{i}-s_{i}}\right|_{r_{i}}
\end{aligned}
$$

where

$$
1 / q_{i}=1 / p+1 / r_{i} \text {. }
$$

Combining the inequalities we find

$$
\left|f_{1} \cdot f_{2} \sigma^{\alpha}\right|_{p} \leqslant C\left|f_{1}\right|_{p, s, 0}\left|f_{2}\right|_{p, s_{1}, 0}\left|f_{2}\right|_{p, s_{2}, 0}\left|\sigma^{t_{1}-s_{1}}\right| r_{1} \times\left|\sigma^{t_{2}-s_{2}}\right|_{r_{2}} .
$$

We need show $r_{i}>0$ and $\left|\sigma^{t_{i}-s_{i}}\right|_{r_{i}}<\infty$.

Now

$$
\begin{aligned}
1 / r_{1} & =1 / p_{1}+s_{1} / n-1 / p \\
& =s_{2} /\left(s_{1}+s_{2}\right) p+s_{1} / n-1 / p=s_{1}\left(1 / n-1 /\left(s_{1}+s_{2}\right) p\right)>0
\end{aligned}
$$

since $\left(s_{1}+s_{2}\right) p>n$.

Using the same reasoning on $r_{2}$ we conclude

$$
r_{i}=\left(s_{1}+s_{2}\right) n p / s_{i}\left(\left(s_{1}+s_{2}\right) p-n\right) .
$$

We need only show $\left(t_{i}-s_{i}\right) r_{i}+n<0$. That is

$$
\left(t_{i}-s_{i}\right)\left(s_{1}+s_{2}\right) n p / s_{i}\left(\left(s+s_{2}\right) p-n\right)+n<0 .
$$

This is equivalent to

$$
\left(t_{i}-s_{i}\right)\left(s_{1}+s_{2}\right) p+s_{i}\left(\left(s_{1}+s_{2}\right) p-n\right)<0 .
$$


This reduces to checking

$$
t_{i} p-n<0 .
$$

But, by construction $t_{i}<\alpha<n / p$. Q.E.D.

Theorem 5.6. Let $p>1$ and $s>n / p$ and $0 \leqslant t \leqslant s$. Then pointwise multiplication induces a continuous map from $M_{s, \delta_{1}}^{p} \times M_{t, \delta_{2}}^{p} \rightarrow M_{t, \delta_{1}+\delta_{2}+\alpha}^{p}$ where $\alpha<n / p$.

Proof. It is sufficient to show that for $|\beta| \leqslant t,(f, g) \rightarrow D^{\beta}(f \cdot g)$ is a continuous map into $M_{0, \delta_{1}+\delta_{2}+\alpha+\beta}^{p}$. However

$$
D^{\beta}(f \cdot g)=\sum_{\gamma<\beta}\left(\begin{array}{c}
\beta \\
\gamma
\end{array}\right) D^{\gamma} f D^{\beta-\gamma}(g)
$$

and $D^{\gamma} f \in M_{s-\gamma, \delta_{1}+\gamma}^{p}, D^{\beta-\gamma}(g) \in M_{t-\beta+\gamma, \delta_{2}+\beta-\gamma}^{p}$. And so the result follows by the previous lemma (since $s-\gamma+(t-\beta+\gamma)=s+t-\beta \geqslant s>n / p$ ). Q.E.D.

COROLlary 5.7. If $s>n / p$ and $\delta>-n / p$ then $M_{s, \delta}^{p}$ is a continuous ring under pointwise addition and multiplication.

Proof. If follows from the previous lemma setting $\delta_{1}=\delta_{2}=\delta$. Then we have that multiplication is continuous into $M_{s, 2 \delta+\alpha}^{p}$. We need $2 \delta+\alpha \geqslant \delta$. This requires $-\delta \leqslant \alpha<n / p$. Q.E.D.

We do not use the following theorem, but do include it for completeness.

THEOREM 5.8. Let $p>1$, then if $s_{1}>s_{2}$ and $\delta_{1}>\delta_{2}$ then $M_{s_{1}, \delta_{1}}^{p}$ is embedded compactly in $M_{s_{2}, \delta_{2}}^{p}$.

This is found in [20].

TheOREM 5.9. Let $p>1, s \in \mathbf{N}$ and $\delta \in \mathbf{R}$; then $M_{s, \delta}^{p}$ is a reflexive Banach space.

The proof of this is routine.

6. Elliptic operators on weighted Sobolev spaces. We now turn to the properties of elliptic operators as maps between $M_{s, \delta}^{p}$ spaces. We are chiefly concerned with the sort of operators that arise in the proof of decomposition theorems. It turns out that in many cases these operators are homogeneous at infinity. Let us consider an example.

Suppose we wish to find a Banach space $\mathbf{X}$ of vector fields on $\mathbf{R}^{3}$ with the property that it splits into gradient fields and divergent free fields. In order to apply Lemma 2.2 we need to find Banach spaces $S_{1}$ and $S_{2}$ such that grad: $S_{1} \rightarrow \mathrm{X}$, and div: $\mathrm{X} \rightarrow S_{2}$ with the property that for $\Delta: S_{1} \rightarrow S_{2}, \operatorname{ker}(\Delta)=$ $\operatorname{ker}(\mathrm{grad})$ and $\operatorname{Rng}(\Delta)=\operatorname{Rng}($ div $)$.

A natural choice of spaces $\mathcal{S}_{1}, \mathbf{X}$, and $\delta_{2}$ would seem to be Sobolev spaces $W^{p, s+1}, W^{p, s}$ and $W^{p, s-1}$ respectively. However for every choice of $p$ and $s$ it 
is not the case that $\operatorname{Rng}(\Delta)=\operatorname{Rng}($ div). To see this let us work in spherical coordinates $(r, \theta), \theta \in S^{n-1}$. Then we have for any $\delta \in \mathbf{R}$ that

$$
\operatorname{div}\left(\sigma^{\delta}\right)=\frac{1}{r^{n-1}} \frac{\partial}{\partial r} r^{n-1}\left(1+r^{2}\right)^{\delta / 2}=\frac{(n-1) \sigma^{\delta}}{r}+\frac{\delta}{2} \sigma^{\delta-2}(2 r) .
$$

On the other hand if $\Delta w=\operatorname{div}\left(\sigma^{\delta}\right)$ then $\partial w / \partial r=\sigma^{\delta}$. Thus we know $w(r, \theta)=\int_{1}^{r}\left(1+t^{2}\right)^{\delta / 2} d t+C_{0}$.

Assuming $\delta<0, \delta \neq-1$ an easy application of Lemma 5.1 yields positive numbers $C_{1}$ and $C_{2}$ such that

$$
\frac{C_{1} r^{\delta+1}}{\delta+1}<w(r, \theta)<C_{2} \frac{r^{\delta+1}}{\delta+1}
$$

(If $w \rightarrow 0$ as $r \rightarrow \infty$ then $C=0$.) If $\delta+1>0$ then clearly $w \notin L^{p}$ and we are done. If $\delta+1<0$, then $|w(r, \theta)|>C_{2} r^{\delta+1} /|\delta+1|$. Now let $-1-n / p$ $<\delta<-n / p+1$. Then it is easy to check that $\operatorname{div}\left(\sigma^{\delta}\right) \in W^{p, s}$ for all $s$ and $w \notin L^{p}$.

Now given $p$, for what range of $\delta$ would one expect $\Delta: M_{s, \delta}^{p} \rightarrow M_{s-2, \delta+2}^{p}$ to be an isomorphism? First of all $\delta$ must be sufficiently large so as to preclude constants in $M_{s, \delta}^{p}$. As we have seen in the previous section, one needs $\delta>-n / p$. On the other hand, $\delta$ must not be too large. Since $C_{0}^{\infty} \subset M_{s-2, \delta+2}^{p}$ we know $M_{s, \delta}^{p}$ must contain the inverse image of $C_{0}^{\infty}$ under the Laplacian. Since $C_{n}|x|^{2-n}\left(C_{n}=\pi^{n / 2} 2^{n} / \Gamma(n / 2-1)\right)$ is the fundamental solution for $\Delta$, one can show if $\Delta f \in C_{0}^{\infty}$ and $f \in M_{s, \delta}^{p}$ then

$$
f(x)=C \int_{\mathbf{R}^{n}} \frac{g(y)}{|x-y|^{n-2}} d V .
$$

At infinity $f$ " behaves like" $|x|^{2-n}$ and so $\delta$ must not be so large to disallow such functions. Thus one must have $\sigma^{2-n} \in M_{s, \delta}^{p}$. This requires $\delta<-2+$ $n(1-1 / p)$.

Heuristically then, one would expect $\Delta: M_{s, \delta}^{p} \rightarrow M_{s-2, \delta+2}^{p}$ to be an isomorphism for $-n / p<\delta<-2+n(1-1 / p)$. This is in fact the case. We will generalize this result to operators which are homogeneous at infinity.

Definition 6.1. Let $A$ be a linear differential operator. We say $A$ is asymptotically homogeneous of degree $(p, s, \delta)$ if there exists a homogeneous differential operator $A_{\infty}$ with constant coefficients such that when $A-A_{\infty}$ is considered as a map between $M_{s, \delta}^{p}\left(|x| \geqslant R, R^{k}\right) \rightarrow M_{s-2, \delta+2}^{p}\left(|x| \geqslant R, R^{k}\right)$ with operator norm $\left\|A-A_{\infty}\right\|_{p, s, \delta, R}$ we have $\lim _{R \rightarrow \infty}\left\|A-A_{\infty}\right\|_{p, s, \delta, R}=0$.

Definition 6.2. Given $p, s, n$, and $m$, we say $\delta$ is exceptional if $\delta+n / p$ or $\delta+m-n(1-1 / p)$ belongs to the positive integers.

Recall $A^{*}$ is the formal adjoint of $A$.

THEOREM 6.3. Let $p>1$, and $A=\sum_{|\alpha| \leqslant m} a_{\alpha} D^{\alpha}$ be a uniformly elliptic mth order operator. Suppose $s \geqslant m$ and each $a_{\alpha}$ is locally of class $W^{p, S_{|\alpha|}, S_{k}>n / p}$ $-m+|\alpha|+1$ and that for $\delta$ not exceptional we have $A$ is asymptotically 
homogeneous of degree $(p, s, \delta)$. Then as a map from $M_{s, \delta}^{p}\left(\mathbf{R}^{n, k}\right) \rightarrow$ $M_{s-m, \delta+m}^{p}\left(\mathbf{R}^{n}, \mathbf{R}^{l}\right)$ we have

(1) $A$ has finite-dimensional kernel and closed range.

(2) The range of $A$ equals $\left\{f \in M_{s-m, \delta+m}^{p}: \int f \cdot h d V=0\right.$ for all $h \in$ $M_{0,-(\delta+m)}^{p^{\prime}}$ such that $\left.A^{*}(h)=0\right\}$.

Proof. We break the proof into several steps.

Step 1. This theorem holds for $A_{\infty}$. This is done for $A_{\infty}=\Delta^{m}$ in some detail in [22]. As is remarked there the reasoning in that paper extends without difficulty to general elliptic homogeneous operators with constant coefficients.

Step 2. $A$ has finite-dimensional kernel. It follows from Step 1 that we may write $M_{s, \delta}^{p}=\operatorname{ker}\left(A_{\infty}\right) \oplus W$ and that there is a $C_{A}>0$ such that for every $f \in W$

$$
|f|_{p, s, \delta} \leqslant C_{A}\left|A_{\infty} f\right|_{p, s-m, \delta+m}
$$

Claim. $\operatorname{ker}(A) \cap W$ is finite dimensional.

To establish this claim we show if $\left\{f_{i}\right\}$ is a sequence in $\operatorname{ker}(A) \cap W$ with $\left|f_{i}\right|_{p, s, \delta}=1$ then $f_{i}$ has a convergent subsequence. (This proof is a modification of Theorem 4.1 of [24].) Let $\varphi_{k}$ be a $C^{\infty}$ function such that $\left|\varphi_{k}\right|<1$ and $\varphi_{k}(x)=1$ for $|x| \leqslant k$ and $\varphi_{k}(x)=0$ for $|x| \geqslant 2 R$. For any $u \in \operatorname{ker}(A) \cap W$ we have

$$
\begin{aligned}
|u|_{p, s, \delta} & \leqslant C\left|A_{\infty} u\right|_{p, s-m, \delta+m} \\
& \leqslant C\left(\left|A_{\infty}\left(\varphi_{R} u\right)\right|_{p, s-m, \delta+m}+\left|A_{\infty}\left(1-\varphi_{k}\right) u\right|_{p, s-m, \delta+m}\right) .
\end{aligned}
$$

Also

$$
\left|A_{\infty}\left(\varphi_{R} u\right)\right|_{p, s-m, \delta+m} \leqslant C\left|\varphi_{R} u\right|_{p, s, \delta} .
$$

Now $\varphi_{R} u$ has compact support in $\{x:|x| \leqslant 2 R\}$, and in fact there is a constant depending only on $R, C_{R}$, such that

$$
\left|\varphi_{R} u\right|_{p, s, \delta} \leqslant C_{R}|u|_{p, s}
$$

where ||$_{p, s}$ is the Sobolev norm on $W^{p, s}\left(B_{2 R}\right)$. Now, using Theorem 3.6 we find

$$
\left|\varphi_{R} u\right|_{p, s, \delta} \leqslant C\left(|A u|_{p, s-m}+|u|_{p, 0}\right) \text {. }
$$

Applying this inequality to $f_{i}$ we find

$$
\left|\varphi_{R} f_{i}\right|_{p, s, \delta} \leqslant C\left|f_{i}\right|_{p, 0} .
$$

We now may apply the Rellich Compactness Theorem (Theorem 3.1) to $\left\{f_{i}\right\}$ as a bounded sequence in $W^{p, s}\left(B_{2 R}\right)$. So by passing to a subsequence we may assume $\left\{f_{i}\right\}$ converges in $L^{p}\left(B_{2 R}\right)$. Substituting $f_{i}-f_{j}$ into the above inequality we find $\left\{\varphi_{R} f_{i}\right\}$ is Cauchy in $M_{s, \delta}^{p}$. Thus $\left\{A_{\infty}\left(\varphi_{R} f_{i}\right)\right\}$ is Cauchy in $M_{s-m, \delta+m}^{p}$. 
The second term in the inequality is handled as follows.

$$
\begin{aligned}
&\left|A_{\infty}\left(1-\varphi_{R}\right) u\right|_{p, s-m, \delta+m} \leqslant\left|\left(1-\varphi_{R}\right) A_{\infty} u\right|_{p, s-m, \delta+m} \\
&+\left|\left(1-\varphi_{R}\right) A_{\infty} u-A_{\infty}\left(1-\varphi_{R}\right) u\right|_{p, s-m, \delta+m} \\
& \leqslant \mid(1-\left.\varphi_{R}\right)\left.\left(A_{\infty} u-A u\right)\right|_{p, s-m, \delta+m} \\
&+\left|\left(1-\varphi_{R}\right) A_{\infty} u-A_{\infty}\left(1-\varphi_{R}\right) u\right|_{p, s-m, \delta+m} \\
& \leqslant \mid\left(A_{\infty}\right.-A)\left.\left(1-\varphi_{R}\right) u\right|_{p, s-m, \delta+m} \\
&+\left|\left(1-\varphi_{R}\right)\left(A_{\infty} u-A u\right)-\left(A_{\infty}-A\right)\left(1-\varphi_{R}\right) u\right|_{p, s-m, \delta+m} \\
&+\left|\left(1-\varphi_{R}\right) A_{\infty} u-A_{\infty}\left(1-\varphi_{R}\right) u\right|_{p, s-m, \delta+m} \\
& \leqslant \| A_{\infty}-A \|_{p, s, \delta, R}\left|\left(1-\varphi_{R}\right) u\right|_{p, s, \delta} \\
&+|\cdots| p, \ldots-m, \delta+m+|\cdots|_{p, s-m, \delta+m} .
\end{aligned}
$$

Now there is a constant $M$ such that for $R$

$$
\begin{aligned}
\left|\left(1-\varphi_{R}\right) u\right|_{p, s, \delta} & \leqslant M|u|_{p, s, \delta} \leqslant M C_{A}\left|A_{\infty} u\right|_{p, s-m, \delta+m} \\
& \leqslant M C_{A}\left(\left|A_{\infty}\left(1-\varphi_{R}\right) u\right|_{p, s-m, \delta+m}+\left|A_{\infty}\left(\varphi_{R} u\right)\right|_{p, s-m, \delta+m}\right) .
\end{aligned}
$$

Substituting (52) into inequality (51) we get

$$
\begin{aligned}
\left|A_{\infty}\left(1-\varphi_{R}\right) u\right|_{p, s-m, \delta+m} \leqslant\left\|A_{\infty}-A\right\|_{p, s, \delta, R}(M C)\left|A_{\infty}\left(1-\varphi_{R}\right) u\right|_{p, s-m, \delta+m} \\
+M C\left|A_{\infty}\left(\varphi_{R} u\right)\right|_{p, s-m, \delta+m}+|\cdots|_{p, s-m, \delta+m} \\
+|\cdots|_{p, s-m, \delta+m} .
\end{aligned}
$$

Pick $R$ sufficiently large so that $M C\left\|A_{\infty}-A\right\|_{p, s, \delta, R}<1$. Then after subtraction and division one obtains for each $f_{i}$

$$
\begin{aligned}
\mid A_{\infty}\left(\left(1-\varphi_{R}\right)\right. & \left.f_{i}\right)\left.\right|_{p, s-m, \delta+m} \leqslant D\left(\left|A_{\infty}\left(\varphi_{R} f_{i}\right)\right|_{p, s-m, \delta+m}\right) \\
& +\left|\left(1-\varphi_{R}\right)\left(A_{\infty}-A\right) f_{i}-\left(A_{\infty}-A\right)\left(1-\varphi_{R}\right) f_{i}\right|_{p, s-m, \delta+m} \\
& +\left|\left(1-\varphi_{R}\right) A_{\infty} f_{i}-A_{\infty}\left(1-\varphi_{R}\right) f_{i}\right|_{p, s-m, \delta+m}
\end{aligned}
$$

We already know $\left\{A_{\infty} \varphi_{R} f_{i}\right\}$ is Cauchy.

Each of the sequences

$$
\begin{aligned}
& \left\{\left(1-\varphi_{R}\right)\left(A_{\infty}-A\right) f_{i}-\left(A_{\infty}-A\right)\left(1-\varphi_{R}\right) f_{i}\right\}, \\
& \left\{\left(1-\varphi_{R}\right) A_{\infty} f_{i}-A_{\infty}\left(1-\varphi_{R}\right) f_{i}\right\}
\end{aligned}
$$

consists of functions with compact support and are bounded in $W^{p, s+1}$. (The highest terms cancel.) Hence we may apply the Rellich theorem twice more to conclude that after passing to a subsequence $\left\{A_{\infty}\left(\left(1-\varphi_{R}\right) f_{i}\right)\right\}$ is Cauchy in $M_{s, \delta}^{p}$. Hence $f_{i}$ is Cauchy in $\operatorname{ker}(A) \cap W$ which is closed. Thus the subsequence $\left\{f_{i}\right\}$ converges and we have established the claim. 
Now $\operatorname{ker}(A) \subset \operatorname{ker}(A) \cap W \oplus \operatorname{ker}(A) \cap \operatorname{ker}\left(A_{\infty}\right)$ and so is finite dimensional.

Step 3. $A$ has closed range.

First write $M_{s, \delta}^{p}=\operatorname{ker}(A) \oplus\left(\operatorname{ker}\left(A_{\infty}\right) \theta \operatorname{ker}(A)\right) \oplus W$. The first two summands are finite dimensional and so $W$ is closed. Let $\left\{e_{1}, \ldots, e_{r}\right\}$ span $\left(\operatorname{ker}\left(A_{\infty}\right) \theta \operatorname{ker}(A)\right)$. Note that $\left\{A\left(e_{1}\right), \ldots, A\left(e_{r}\right)\right\}$ are linearly independent (and $\operatorname{sp}\left(A\left(e_{1}\right), \ldots, A\left(e_{r}\right)\right)$ is linearly independent from $A(W)$ ). We may express $M_{s-m, \delta+m}^{p}$ as $\operatorname{sp}\left(A\left(e_{1}\right), \ldots, A\left(e_{r}\right)\right) \oplus Y$ where $A(W) \subset Y$.

We shall show that $f(W)$ is closed in $M_{s-m, \delta+m}^{p}$. Supposing this is true it follows that $f\left(M_{s, \delta}^{p}\right)$ is closed. To see this note

$$
A\left(M_{p, \delta}^{p}\right)=A\left(\operatorname{sp}\left(e_{1}, \ldots, e_{r}\right) \oplus W\right) .
$$

Let $\lim _{i \rightarrow \infty} A\left(\sum_{j=1}^{r} a_{i j} e_{j}+w_{i}\right)=u=\sum b_{l} f\left(e_{l}\right)+y=\sum c_{l} f\left(e_{l}\right)+w$. We may put an equivalent norm || on $M_{s-m, \delta+m}^{p}$ by setting $\left|\sum b_{l} f\left(e_{l}\right)+y\right|_{A}=$ $\max \left(\left|b_{l}\right|,|y|_{p, s-m, \delta+m}\right)$. Then $A\left(\sum_{j=1}^{r} a_{i j} e_{j}+w_{i}\right) \rightarrow u \Rightarrow a_{i j} \rightarrow b_{j}$ for each $j=$ $1, \ldots, r$. Thus $A\left(\sum_{j=1}^{r} a_{i j} e_{j}\right) \rightarrow \sum b_{l} f\left(e_{l}\right)$. It follows immediately that $f\left(w_{i}\right) \rightarrow$ $y$. But if $A(w)$ is closed, then $y=A(\bar{w}), \bar{w} \in W$ and we are done. Thus we need to show

Claim. $A(W)$ is closed in $M_{s-m, \delta+m}^{p}$.

As in the proof of Theorem 3.9 we need only show there is a $C>0$ such that for all $f \in W$,

$$
|f|_{p, s, \delta} \leqslant C|A f|_{p, s-m, \delta+m} .
$$

Suppose no such $C$ exists. Then there is a sequence $\left\{f_{i}\right\} \subset W$ such that $\left|f_{i}\right|_{p, s, \delta}=1$ and $\left|A f_{i}\right|_{p, s-m, \delta+m} \rightarrow 0$. As in the proof of the previous step we know there is a $C_{A}>0$ such that for any $f \in W_{1}$

$$
|f|_{p, s, \delta} \leqslant C_{A}\left|A_{\infty} f\right|_{p, s-m, \delta+m} .
$$

We proceed as in the previous step.

$$
\begin{aligned}
|f|_{p, s, \delta} \leqslant & C_{A}\left|A_{\infty} f-A f\right|_{p, s-m, \delta+m}+|A f|_{p, s-m, \delta+m} \\
\leqslant & C_{A}\left|\left(A_{\infty}-A\right) \varphi_{R} f\right|_{p, s-m, \delta+m} \\
& +\left|\left(A_{\infty}-A\right)\left(-\varphi_{R}\right) f\right|_{p, s-m, \delta+m}+|A f|_{p, s-m, \delta+m} .
\end{aligned}
$$

There is a $C_{1}>0$ such that for all $R$

$$
\left|\left(A_{\infty}-A\right) \varphi_{R} f\right|_{p, s-m, \delta+m} \leqslant C_{1}|f|_{p, s} \leqslant C\left(|A f|_{p, s-m}+|f|_{p}\right)
$$

where as before ||$_{p, k}$ is the norm on $W^{p, k}\left(B_{2 R}\right)$. Note that $|A f|_{p, s-m} \rightarrow 0$ and that $\left\{f_{i}\right\}$ is bounded in $W^{p, s}\left(B_{2 R}\right)$. Hence the compactness argument used before tells us that by passing to a subsequence we may conclude $\left\{\left(A_{\infty}-\right.\right.$ A) $\left.\varphi_{R} f_{i}\right\}$ is Cauchy in $M_{s-m, \delta+m}^{p}$.

We also have

$$
\begin{aligned}
\left|A_{\infty}-A\left(1-\varphi_{R}\right) f\right|_{p, s-m, \delta+m} & \leqslant\left\|A_{\infty}-A\right\|_{p, s, \delta, R}\left|\left(1-\varphi_{R}\right) f\right|_{p, s, \delta} \\
& \leqslant M\left\|A_{\infty}-A\right\|_{p, s, \delta, R}|f|_{p, s, \delta} .
\end{aligned}
$$

Substituting this inequality back into inequality (58), we find by choosing $R$ sufficiently large that 


$$
|f|_{p, s, \delta} \leqslant C\left(\left|\left(A_{\infty}-A\right) \varphi_{R} f\right|_{p, s-m, \delta+m}+|A f|_{p, s-m, \delta+m}\right) .
$$

By assumption $\left\{A f_{i}\right\}$ is Cauchy in $M_{s-m, \delta+m}^{p}$ and so $\left\{f_{i}\right\}$ is Cauchy in $\boldsymbol{M}_{s, \delta}^{p}$. Hence $f_{i} \rightarrow \bar{f} \in M_{s, \delta}^{p}$. But $|\bar{f}|_{p, s, \delta}=1$ and $|A \bar{f}|_{p, s, \delta}=0$. Thus $\bar{f} \in \operatorname{ker}(A) \cap \stackrel{W}{W}$ and $\bar{f} \neq 0$. This is impossible and our inequality is established.

Step 4 (Regularity). There is a $C>0$ such that for all $f \in M_{s, \delta}^{p}$

$$
|f|_{p, s, \delta} \leqslant C\left(|A f|_{p, s-m, \delta+m}+|f|_{p, s-m, \delta}\right) \text {. }
$$

This is proven using the same techniques found in the previous steps. That the inequality holds for $A_{\infty}$ is found in [22].

Step 5. Completion of the proof.

Since $A$ has closed range and $M_{s, \delta}^{p}$ is reflexive we use the same argument as found in proof of Theorem 3.11. Note that making the usual identifications we may set $\left(M_{0, \delta}^{p}\right)^{*}$ to be $M_{0,-\delta}^{p^{\prime}}$ where $1 / p+1 / p^{\prime}=1$.

Now let $s>m$. If $f \in A\left(M_{s, \delta}^{p}\right) \subset\left(M_{m, \delta}^{p}\right)$ it is clear that $f \in\{g \in$ $M_{s-m, \delta+m}^{p}: g, h=0$ for $\left.h \in M_{0,-\delta}^{p^{\prime}}: A^{*}(h)=0\right\}$. Now suppose $f \in M_{s-m, \delta+m}^{p}$ and $\int f \cdot h d V=0$. Then we know $f=A(u)$ for some $u \in M_{m, \delta}^{p}$. However we see from Step 4 that in fact $u \in M_{s, \delta}^{p}$. Q.E.D.

REMARK. It is shown in [22] that even for the Laplacian Theorem 6.3 fails when $\delta$ is exceptional.

It should also be remarked that this theorem may be proven using topological methods based on Theorem 5.8. This approach is used in [20, 22].

Let us give an application of the preceding theorem. This shows our heuristic reasoning is correct.

Proposition 6.4 (McOWen). Let $p>1, s \geqslant 2$ and $\delta$ not exceptional. Then $\Delta: \quad M_{s, \delta}^{p} \rightarrow M_{s-2, \delta+2}^{p}$ is one-one iff $-n / p<\delta$ and onto iff $\delta<-2+$ $n(1-1 / p)$.

Proof. Suppose $\delta>-n / p, f \in M_{0, \delta}^{p}$ and $\Delta f=0$. It follows from the regularity estimate (Step 4 of the above proof) that $f \in M_{s, \delta}^{p}$ for all $s$, and so $f$ is $C^{\infty}$. It follows from Theorem 5.4 that $f(x) \rightarrow 0$ as $|x| \rightarrow \infty$. It follows from the maximum principle that $f=0$. If $\delta<-n / p$ then $1 \in M_{s, \delta}^{p}$ and so $\Delta$ is not one-one.

It follows from Theorem 6.3 that $\Delta$ is onto iff $\left\{f \in M_{0,-(\delta+2)}^{p^{\prime}}: \Delta(f)=0\right\}$ is trivial $\left(1 / p+1 / p^{\prime}=1\right)$. From the above discussion this is equivalent to $-(\delta+2)>-n / p^{\prime}$, i.e. $\delta<-2+n / p^{\prime}$. Q.E.D.

CoRollary 6.5. If $p>1, s \geqslant 2$, and $-n / p<\delta<-2+n(1-1 / p)$, then $\Delta: M_{s, \delta}^{p} \rightarrow M_{s-2, \delta+2}^{p}$ is an isomorphism.

Let us consider the Laplacian in more detail. Suppose $g$ is $C^{\infty}$ and $g=O\left(|x|^{-t}\right)$ on $\mathbf{R}^{3}$ for $t \geqslant 3$. Then $g \in M_{s, \beta}^{p}$ for $\beta>-3 / p+t$. Let $-3 / p<$ $\delta<-2+3(1-1 / p)$. Note $M_{s, \beta}^{p} \subset M_{s, \delta+2}^{p}$ and so we may find a solution to $\Delta f=g$ with $f \in M_{s+2, \delta}^{p}$. Thus in general we may conclude $f=o\left(|x|^{\alpha}\right)$ for $\alpha>-3 / p-\delta>-1$. The question does arise: When may we conclude $f=O\left(|x|^{-t+2}\right)$ ? In this case $g \in \Delta\left(M_{s+2, \beta-2}^{p}\right)$ and so we must have $\int f \cdot h d V=$ 0 for all $h \in M_{0,-\beta^{\prime}}^{p^{\prime}}$, such that $\Delta(h)=0$. If $h \in M_{0,-\beta}^{p^{\prime}}$, and $\Delta(h)=0$ by 
regularity $h \in M_{s,-\beta}^{p}$ for all $s$. Thus we may conclude $h=O\left(|x|^{\gamma}\right)$ for $\gamma>$ $-3 / p^{\prime}+\beta>t-3$. Say $t=k+3$. Then $h$ may be a harmonic polynomial of degree $k$. In this way we recover the classical result that if $\Delta f=O\left(|x|^{-t}\right)$ then $f=O\left(|x|^{2-t}\right)$ iff $\int f p_{k}=0$ for all harmonic polynomials $p_{k}$ of degree $k=t-3$.

Actually the above discussion is not so remarkable. The sort of reasoning given was used by McOwen in his proof of Step 1 of Theorem 6.3 (see [22]).

There are various conditions on $A$ which guarantee that the operator is asymptotically homogeneous of degree $(p, s, \delta)$. One straightforward criterion is the following.

PROPOSITION 6.6. Let $A=\Sigma_{|\alpha| \leqslant m} a_{\alpha} \mathscr{D}^{\alpha}$. Suppose $a_{\alpha} \in C^{s-m}\left(\mathbf{R}^{n}, \mathbf{R}^{n}\right)$. There is an operator $A_{\infty}=\sum_{|\alpha|=m} \bar{a}_{\alpha} \mathscr{O D}^{\alpha}$ with $\bar{a}_{\alpha}$ constant such that for $|\gamma| \leqslant s-m$

$$
\begin{array}{cc}
\limsup _{|x| \rightarrow \infty}\left|D^{\gamma} a_{\alpha} \sigma^{m-|\alpha|+|\gamma|}\right|=0 & \text { for }|\alpha|<m, \\
\limsup _{|x| \rightarrow \infty}\left|D^{\gamma}\left(a_{\alpha}(x)-\bar{a}_{\alpha}\right) \sigma^{|\gamma|}\right|=0 & \text { for }|\alpha|=m .
\end{array}
$$

Then $A$ is asymptotically homogeneous of degree $(p, s, \delta)$ for any $\delta$.

Most applications require weaker hypotheses on the coefficients of $A$. The following proposition is more useful.

Proposition 6.7. Let $A=\sum_{|\alpha| \leqslant m} a_{\alpha} D^{\alpha}$ and $A_{\infty}=\sum_{|\alpha|=m} \bar{a}_{\alpha} D^{\alpha}$, with $\bar{a}_{\alpha}$ constant such that if $B=A-A_{\infty}=\sum b_{\alpha} D^{\alpha}$, then $b_{\alpha} \in M_{t_{\alpha}, l_{\alpha}}^{p}$ with $t_{\alpha}>n / p$ $+|\alpha|-m$ and $l_{\alpha}>m-|\alpha|-n / p$. Then $A$ is asymptotically homogeneous of degree $(p, s, \delta)$ for any $\delta \in \mathbf{R}$.

Proof. Let

$$
|f|_{p, s, \delta, R}=\left(\int_{|x|>R} \sum_{|\alpha| \leqslant s}\left|D^{\alpha} f_{\sigma}^{\delta+|\alpha|}\right|^{p} d V\right)^{1 / p}
$$

Now,

$$
\left|\left(A-A_{\infty}\right) f\right|_{p, s-m, \delta+m, R} \leqslant \sum_{|\alpha|<m}\left|b_{\alpha} D^{\alpha} f\right|_{p, s-m, \delta+m, R}
$$

Also

$$
\begin{aligned}
\left|b_{\alpha} D^{\alpha} f\right|_{p, s-m, \delta+m} & =\sum_{|\gamma|<s-m}\left|D^{\gamma}\left(b_{\alpha} D^{\alpha} f\right)\right|_{p, s, \delta+m+|\gamma|, R} \\
& \leqslant \sum_{|\gamma|<s-m} \sum_{\beta<\gamma}\left(\begin{array}{l}
\beta \\
\gamma
\end{array}\right)\left|D^{\beta} b_{\alpha} D^{\alpha+\gamma-\beta} f\right|_{p, s, \delta+m+|\gamma|, R} .
\end{aligned}
$$

By assumption $D^{\alpha+\gamma-\beta} f \in M_{s+|\beta|-|\gamma|-|\alpha|, \delta+|\alpha|+|\gamma|-|\beta|}^{p}$ and $D^{\beta} b_{\alpha} \in$ $M_{t_{\alpha}-\beta, l_{\alpha}+\beta}^{p}$.Thus using Theorem 5.6 (noting the theorem works over spaces of functions over exteriors of spheres) we get

$$
\begin{gathered}
\left|D^{\beta} b_{\alpha} D^{\alpha+\gamma-\beta} f\right|_{p, 0, m+|\gamma|+\delta, R} \\
\leqslant C\left|D^{\beta} b_{\alpha}\right|_{p, t_{\alpha}-|\beta|, l_{\alpha}+|\beta|, R}\left|D^{\alpha+\gamma-\beta} f\right|_{p, s+|\beta|-|\gamma|-|\alpha|, \delta+|\alpha|+|\gamma|-|\beta|, R}
\end{gathered}
$$


if

(1) $t_{\alpha}-|\beta|+(s+|\beta|-|\gamma|-|\alpha|)>n / p$ and

(2) $l_{2}+|\beta|+(\delta+|\alpha|+|\gamma|-|\beta|) m+|\gamma|+\delta-n / p$.

Now (1) is equivalent to $t_{\alpha}>n / p+\gamma+|\alpha|-s$. But by assumption $t_{\alpha}>$ $n / p+|\alpha|-m$ and $|\gamma| \leqslant s-m$. Hence (1) holds.

Again by assumption $l_{\alpha}>m-|\alpha|-n / p$ which implies (2). Thus we have

$$
\left|\left(A-A_{\infty}\right) f\right|_{p, s-m, \delta+m, R} \leqslant C \sum_{|\alpha|<m}\left|b_{\alpha}\right|_{p, t_{\alpha}, l_{\alpha}, R}|f|_{p, s, \delta, R}
$$

and so

$$
\left\|A-A_{\infty}\right\|_{p, s, \delta, R} \leqslant C \sum_{|\alpha|<m}\left|b_{\alpha}\right|_{p, t_{\alpha}, l_{\alpha}, R}
$$

But

$$
\lim _{R \rightarrow \infty}\left|b_{\alpha}\right|_{p, t_{\alpha}, l_{\alpha}, R}=0 \text {. Q.E.D. }
$$

7. Decomposition of tensor fields over asymptotically Euclidean manifolds. That the results of the previous section extend to manifolds with more than one "infinity" is rather easy to see. In fact, after some technical material is given the proofs of the previous section go through with little modification.

The interest in such manifolds stems chiefly from their application in general relativity. They are a natural setting for $n$-body problems.

Definition 7.1. A $n$-manifold $S$ is said to have ends if the complement of a compact set $N_{0}$ in $S$ may be written as the disjoint union $M-N_{0}=$ $\cup_{i=1}^{m} N_{i}$ where each $N_{i}, 1 \leqslant i \leqslant n$, is diffeomorphic under $\varphi_{i}$ to the complement of a unit sphere in $\mathbf{R}^{n}$. Each $N_{i}$ for $1 \leqslant i \leqslant m$ is called an end.

DEFINITION 7.2. A Riemannian manifold $(S, g)$ is called asymptotically Euclidean of degree $(p, s, \delta)$ if on each end there is a coordinate system such that with respect to these coordinates $g_{i j}-\delta_{i j} \in M_{s, \delta}^{p}$. The coordinates are called straightening.

The metric $g$ can be used to give a coordinate free definition of $M_{s, \delta}^{p}(S)$. Let $\langle,\rangle_{x}$ be the inner product on $T_{x} M$ induced by $g$. For $V_{x} \in T_{x} M$, $\left|V_{x}\right|_{x}=\sqrt{\left\langle V_{x}, V_{x}\right\rangle_{x}}$. Also denote the canonical volume form on $S$ by $\mu_{g}$. The geodesic distance from $x$ to $y$ is $d(x, y)$.

Proposition 7.3. Let $(S, g)$ be asymptotically Euclidean of degree $(p, s, \delta)$ with $s>n / p+1$ and $\delta>-n / p$. Then on each end, if $t \leqslant s$, the $M_{t, \beta}^{p}$ norm of any section with respect to straightening coordinates is equivalent to the intrinsic norm

$$
\sum_{|\alpha|<t}\left(\int_{N_{i}}\left|\left(\nabla^{\alpha} f\right) \bar{\sigma}^{\beta+\beta}\right|^{p} \mu_{g}\right)^{1 / p}
$$

where $\bar{\sigma}(x)=\left(1+d\left(x, x_{0}\right)^{2}\right)^{1 / 2}$ for some $x_{0} \in N_{i}$.

Proof. Recall that locally $\mu_{\mathrm{g}}=\sqrt{\operatorname{det} g_{i j}} d x^{1} \ldots d x^{n}$. Since $\sqrt{\operatorname{det} g_{i j}}$ is continuous, everywhere positive and approaches 1 at each infinity, we know, 
given some system of straightening coordinates, there exists constants $C_{1}, C_{2}>0$ such that $0<C_{1}<\sqrt{\operatorname{det} g_{i j}(x)}<C_{2}<0$ for all $x \in S$. The result then follows easily from coordinate formulas for $\nabla^{\alpha}$ and Theorem 5.6. Bounds for $\bar{\sigma} / \sigma$ are obtained using comparison theorems. By assumption the Christoffel symbols are Hölder continuous. Q.E.D.

The definitions and results of the previous two sections extend in a natural way to asymptotically Eulcidean manifolds. In fact we have the following theorem.

THEOREM 7.4. Let $(S, g)$ be an asymptotically Euclidean manifold of degree $(p, t, \beta)$ where $t>n / p+1$ and $\beta>-n / p$. Also let $s \leqslant t$ and $A$ be an mth order uniformly elliptic differential operator between sections of tensor bundles $E$ and $F$ over $S$. Suppose further that with respect to straightening coordinates $A$ may be written as $A=\sum a_{\alpha} D^{\alpha}$ with $a_{\alpha}$ locally in $W^{p,|\alpha|}, s_{|\alpha|}>n / p-m+|\alpha|$ +1 . Then if on each end $A$ is asymptotically homogeneous of degree $(p, s, \delta)$ with $s \leqslant t$ and $\delta$ not exceptional, we have for $A: M_{s, \delta}^{p}(E) \rightarrow M_{s-m, \delta+m}^{p}(F)$ that

(1) $A$ has finite-dimensional kernel and closed range.

(2) The range of $A$ equals $\left\{f \in M_{s-m, \delta+m}^{p}: \int_{S}\langle f, h\rangle_{F} \mu_{g}=0\right.$ for all $h \in$ $M_{0,-(\delta+m)}^{p^{\prime}}$ such that $\left.A^{*}(h)=0\right\}$.

Proof. The proof of this theorem is a straightforward modification of the proof of Theorem 6.3. Simply pick $\varphi_{R, i}, i=1, m$, to be a $C^{\infty}$ function whose support is contained in $N_{i}$. Also let each $\varphi_{R, i}$ be bounded by $1, \varphi_{R, i}(x)=1$ if $\left|\varphi_{i}(x)\right|>R$. Let $\varphi_{0, R}=1-\sum_{i=1}^{m} \varphi_{R, i}$. Using $\varphi_{i, R}$ for $i=0, \ldots, m$ in the place of $\varphi_{R}$ and $\left(1-\varphi_{R}\right)$ the proof of Theorem 6.3 extends to this case. Q.E.D.

REMARK. This proof uses the fact that the tensor bundles inherit asymptotically Euclidean norms from TS. One could define an asymptotically Euclidean bundle and generalize the above results.

We now give some applications of Theorem 7.4. Recall the Laplacian with respect to $g, \Delta_{g}$ is locally given by

$$
\sum_{i, j}(\sqrt{g})^{-1} \frac{\partial}{\partial x^{i}}\left(\sqrt{g} g_{i j} \frac{\partial}{\partial x^{j}}\right) \text { where } g=\operatorname{det} g_{i j} \text {. }
$$

Proposition 7.5. Suppose $p>1, t>n / p+1$ and $(S, g)$ is asymptotically Euclidean of class $(p, t, \beta)$ for $\beta>-n / p$. Then if $-n / p<\delta<-2+$ $n(1-1 / p)$ and $2 \leqslant s \leqslant t$ the Laplacian with respect to $g$ is an isomorphism from $M_{t, \delta}^{p}$ to $M_{t-2, \delta+2}^{p}$.

This is proven similarly to Proposition 6.4 above.

In what follows $\nabla_{g}$ and $\operatorname{div}_{g}$ are the gradient and divergence operators associated with the metric $g$. In coordinates

$$
\nabla_{g} f=\sum_{i} \sum_{j} g_{i j} \frac{\partial f}{\partial x^{j}} \frac{\partial}{\partial x^{i}}
$$


and

$$
\operatorname{div}_{g}\left(\sum V^{i} \frac{\partial}{\partial x^{i}}\right)=\frac{1}{\sqrt{\operatorname{det} g}} \sum_{i, j} \frac{\partial}{\partial x^{i}}\left(\sqrt{\operatorname{det} g} g_{i j} V^{j}\right)
$$

$\operatorname{Div}_{g}$ is $L^{2}$ adjoint of $\nabla_{g}$ using the inner product and volume density associated with $g$. Also $\Delta_{g}=\operatorname{div}_{g} \circ \operatorname{div}_{g}$.

Theorem 7.6 (Helmholtz Decomposition). Suppose $p>1$ and $(S, g)$ is asymptotically Euclidean of class $(p, t, \beta)$ for $t>s / p+1, \beta>-s / p$. Then if $1 \leqslant s \leqslant t-1$ and $-s / p<\delta<-1+n(1-1 / p)$ ( $\delta$ not exceptional $)$, we have

$$
M_{s, \delta}^{p}(T S)=\nabla_{g}\left(M_{s+1, \delta-1}^{p}\right) \oplus \operatorname{ker}\left(\operatorname{div}_{g}\right)
$$

with the range of $\nabla_{g}$ closed. Furthermore if $s>n / p$ the decomposition holds for $-n / p<\delta<n(1-1 / p)$.

Proof. We need show that $\Delta_{g}=\operatorname{div}_{g} \circ \nabla_{g}: M_{s+1, \delta-1}^{p} \rightarrow M_{s-1, \delta+1}^{p}$ satisfies the hypotheses of Lemma 2.2.

Claim. $\operatorname{Ker}\left(\Delta_{g}\right)=\operatorname{ker}\left(\nabla_{g}\right)$.

By restricting our attention to one end at a time we may treat $\Delta_{g}$ as an asymptotically homogeneous operator of degree $(p, t, \delta)$ on $\mathbf{R}^{n}$. Clearly $\operatorname{ker}\left(\nabla_{g}\right) \subset \operatorname{ker}\left(\Delta_{g}\right)$. So suppose $\Delta_{g} u=0$ and $u \in M_{s+1, \delta-1}^{p}$. Since $\Delta_{g} u=0$ using the regularity estimate in the proof of Theorem 6.3 we see $u \in M_{t, \delta-1}^{p}$ and so $u$ is locally $C^{1}$. Also it follows from Theorem 5.1 that $\left|u \sigma^{\beta}(x)\right| \rightarrow 0$ as $|x| \rightarrow \infty$ for all $\beta<(\delta-1)+n / p$. By assumption we may write $\delta=-n / p$ $+3 \varepsilon$ for some $\varepsilon>0$. Then let $\beta=-1+2 \varepsilon$ to conclude that $|u(x)| \leqslant$ $C\left|\sigma^{1-\varepsilon}\right|$ for some $\varepsilon>0$. Now let $x_{0} \in \mathbf{R}^{n}$. We may without loss of generality take $x_{0}=0$. A standard elliptic estimate (see Protter and Weinberger [27]) tells us

$$
|\nabla u(0)| \leqslant \frac{c}{r}\left(M_{r}-m_{r}\right)
$$

where $M_{r}=\max \{u(x):|x|<r\}$ and $m_{r}=\min \{u(x):|x|<r\}$. We know $M_{R}-m_{r}<C r^{1-\varepsilon}$ and so $|\nabla u(0)|<C r^{-\varepsilon}$ for all $r$. Hence as letting $r \rightarrow \infty$, we have $|\nabla u(0)|=0$. It follows $u\left(x_{0}\right)=0$ for all $x_{0} \in R^{n}$ and so $u$ is constant. Hence $\nabla_{g} u=0$.

Claim. $\operatorname{Rng}\left(\Delta_{g}\right)=\operatorname{Rng}($ div $)$.

Clearly $\operatorname{Rng}\left(\Delta_{g}\right) \subset \operatorname{Rng}\left(\operatorname{div}_{g}\right)$. We need $\operatorname{Rng}\left(\operatorname{div}_{g}\right) \subset \operatorname{Rng}\left(\Delta_{g}\right)$. Suppose $\delta<$ $-1+n(1-1 / p)$. Then using Theorem 7.5 we see $\Delta_{g}$ maps $M_{s+1, \delta-1}^{p}$ onto $M_{s-1, \delta+1}^{p}$ and we are done.

Now suppose $s>n / p,-1+n\left(1-1 / p^{\prime}\right)<\delta<n\left(1-1 / p^{\prime}\right)$, and $V=$ $\operatorname{div}_{g} u$ and $u \subset M_{s, \delta}^{p}$. For $r>0, \int_{|x| \leqslant r} \operatorname{div}_{g} u d \mu_{g}=\int_{|x|=r} g(u, n) d S$ where $n$ is normal to $\{|x|=r\}$ with respect to $g$. Now $\left|\int_{|x|=r} g(u, n) d S\right| \leqslant$ $C \int_{|x|=r \mid}|u| r^{n-1} d S_{\theta}$ where $d S_{\theta}$ is the measure on $\{|x|=r\}$ with respect to the Euclidean norm.

Using Theorem 5.6 we have $\left|u(x) \sigma^{\beta}(x)\right| \rightarrow 0$ as $|x| \rightarrow \infty$ for $\beta<\delta+n / p$. We may write $\delta=-1+n(1-1 / p)+3 \varepsilon$ for some $\varepsilon>0$. Thus we may take 
$\beta=n-1+2 \varepsilon$. Hence $|u(x)| \leqslant C|x|^{1-n-\varepsilon}$ for large $|x|$, and so

$$
\left|\int_{|x|=r} g(u, n) d S\right| \leqslant C \int_{|x|=r} r^{1-n-\varepsilon} \cdot r^{n-1} d S_{\varepsilon} \leqslant C r^{-\varepsilon} .
$$

Thus letting $r \rightarrow \infty$, we see

$$
\int_{M} \operatorname{div}_{g}(u) d \mu_{g}=0 .
$$

Hence $\operatorname{Rng}\left(\operatorname{div}_{g}\right) \subset\left\{v \in M_{s-1, \delta+1}^{p}: \int \operatorname{cvd} \mu=0\right.$ for $c$ any constant $\}$. Recall $\operatorname{Rng}\left(\Delta_{g}\right)=\left\{v \in M_{s-1, \delta+1}^{p}: \quad\langle h, v\rangle \mu_{g}=0\right.$ for $\left.h \in M_{0,-(\delta+1)}^{p^{\prime}}, \Delta_{g}(h)=0\right\}$. Now since $\delta+1<1+n / p^{\prime}$, we have $-(\delta+1)>-1-n / p^{\prime}$. Hence using the reasoning of the previous claim if $h \in M_{0,-(\delta+1)}^{p}$ and $\Delta_{g}(h)=0, h$ is constant. Thus $\operatorname{Rng}\left(\operatorname{div}_{g}\right) \subset \operatorname{Rng}\left(\Delta_{g}\right)$. Q.E.D.

Let us continue with this example. The bounds $-n / p<\delta<n /(1-1 / p)$ are sharp for the Helmholtz decomposition to hold. To see this we consider the case where $M=\mathbf{R}^{n}$ and $g$ is the Euclidean metric. Suppose $\delta<-n / p$ $\left(\delta=-n / p\right.$ is exceptional). Then if $L: \mathbf{R}^{n} \rightarrow R$ is linear $L \in M_{s+1, \delta-1}^{p}$. To see this note $|L(x)| \leqslant C|x|$ and so $\left|L(x) \sigma^{\delta-1}(x)\right|_{p} \leqslant C\left|\sigma^{\delta}(x)\right|_{p}$. But since $\delta<$ $-n / p, \delta p+n<0$ and so $\left|\sigma^{\delta}(x)\right| p<\infty$. Thus $\operatorname{ker}(\Delta) \subset M_{s-1, \delta+1}^{p}$ contains all the linear functions. On the other hand, $\operatorname{ker}\left(\nabla_{g}\right)$ contains only the constants.

Now suppose $\delta>n(1-1 / p)$. Then the range of $\Delta$ equals the range of div. Then since $\Delta$ has closed range we know the range of div must be closed. Hence its range must be $\left\{f \in M_{s-1, \delta+1}^{p}: \int f h d v=0\right.$ for $h \in M_{0-(\delta+1)}^{p^{\prime}}$, $\operatorname{grad} h$ $=0\}$. By assumption $-(\delta+1)<-n / p^{\prime}-1$. Thus $M_{0,-(\delta+1)}^{p^{\prime}}$ contains linear functions. Using the characterization of the range of $\Delta$, we find $\operatorname{Rng}(\nabla)^{\perp}$ contains only constants, but $\operatorname{Rng}(\Delta)^{\perp}$ contains linear functions. Thus $\operatorname{Rng}(\Delta)$ cannot equal Rng(div).

Thus if $\delta<-n / p$ or $\delta>n(1-1 / p)$ one of the necessary conditions of Lemma 2.2 is violated.

\section{BIBLIOGRAPHY}

The following bibliography is far from complete. Representative articles from the various subjects touched in our discussion are given. The reader is advised to consult the references in the listed articles.

1. R. Abraham and J. Marsden, Foundations of mechanics, Benjamin, New York, 1978.

2. S. Agmon, A. Douglis and L. Nirenberg, Estimates near the boundary for solutions of elliptic partial differential equations satisfying general boundary conditions. I, Comm. Pure Appl. Math. 12 (1959), 623-727.

3. J. Arms, A. Fischer and J. Marsden, Une approche symplectique pour des théorèmes de decomposition en géometrie ou relativité generale, C.R. Acad. Sci. Paris Sér. A 281 (1975), 517-520.

4. M. Berger and D. Ebin, Some decompositions of the space of symmetric tensors of a Riemannian manifold, J. Differential Geom. 3 (1969), 379-392.

5. J. P. Bourguignon, D. Ebin and J. Marsden, Sur le noyau des operateurs pseudo-differential a symbole surjectif et non injectif, C.R. Acad. Sci. Paris Sér. A 282 (1975), 867-870.

6. M. Cantor, The existence of non-trivial asymptotically flat initial date for vacuum spacetimes, Comm. Math. Phys. 57 (1977), 83-96.

7. , On the existence of asymptotically flat initial data sets for spacetimes containing manifolds with ends, preprint, August, 1979. 
8. _ A necessary and sufficient condition for York data to specify an asymptotically flat spacetime, J. Math. Phys. 20 (1979), 1741-1744.

9. __ Perfect fluid flows over $\mathbf{R}^{n}$ with asymptotic conditions, J. Funct. Anal. 18 (1975), 73-84.

10. Some problems of global analysis on asymptotically simple manifolds, Compositio Math. 38 (1979), 3-35.

11. , Spaces of functions with asymptotic conditions, Indiana Univ. Math. J. 24 (1974), 897-902.

12. M. Cantor and D. Brill, The Laplacian on asymptotically flat manifolds and the specification of scalar curvature, Compositio Math. (to appear).

13. Y. Choquet-Bruhat and D. Christodoulou, Elliptic systems in Hilbert spaces on manifolds which are euclidean at infinity, Acta. Math. 146 (1981), 129-150. (Also see C.R. Acad. Sci. Paris Sér. A-B 290 (1980), 781-785.)

14. D. Christodoulou, The boost problem for weakly coupled quasilinear hyperbolic systems of the second order, preprint, February, 1980.

15. D. Ebin, The manifold of Riemannian metrics, Proc. Sympos. Pure Math., vol. 15, Amer. Math. Soc., Providence, R.I., 1970, pp. 11-40.

16. A. Fischer and J. Marsden, The initial value problem and the dynamical formulation of general relativity, General Relativity (S. Hawking and W. Israel, eds.), Cambridge Univ. Press, New York and London, 1979, pp. 138-211.

17. L_ Linearization stability of nonlinear partial differential equations, Proc. Sympos. Pure Math., vol. 27, Amer. Math. Soc., Providence, R.I., 1975, pp. 219-263.

18. D. Gilbarg and N. S. Trudinger, Elliptic partial differential equations of second order, Springer-Verlag, New York, 1977.

18a. H. Helmholtz, Über Integrale der Hydrodynamischen Gleichungen, welch den Wirbelbewegungen, J. Reine Angew. Math. 55 (1858), 25-55.

18b. V. W. Hodge, Theory and applications of harmonic integrals, 2nd ed., Cambridge Univ. Press, New York and London, 1952.

18c. K. Kodaira, Harmonic fields in Riemannian manifolds, Ann. of Math. (2) 50 (1949), $587-665$.

18d. J. Kohn and L. Nirenberg, Non-coercive boundary value problems, Comm. Pure Appl. Math. 18 (1965), 443-492.

19. S. Lang, Differentiable manifolds, Addison-Wesley, Reading, Mass., 1972.

20. R. Lockhart, Fredholm properties of a class of elliptic operators on noncompact manifolds, preprint, 1979.

21. J. Marsden, Applications of global analysis in mathematical physics, Publish or Perish, Boston, Mass., 1974.

22. R. McOwen, Behavior of the Laplacian on weighted Sobolev spaces, Comm. Pure Appl. Math. 32 (1979), 783-795.

23. On elliptic operators in $\mathbf{R}^{n}$, Comm. Partial Differential Equations (to appear).

23a. C. Morry, Multiple integrals in the calculus of variations, Springer-Verlag, Berlin and New York, 1966.

23b. C. Morry and J. Eells, A variational method in the theory of harmonic integrals, Ann. of Math. (2) 63 (1956), 91-128.

24. L. Nirenberg and H. Walker, Nullspaces of elliptic partial differential operators in $\mathbf{R}^{n}$, J. Math. Anal. Appl. 42 (1973), 271-301.

25. R. Palais, Foundations of global non-linear analysis, Benjamin, New York, 1968.

26. Seminar on the Atiyah-Singer Index Theorem, Ann. of Math. Studies, no. 57, Princeton Univ. Press, Princeton, N.J., 1965.

27. M. Protter and H. Weinberger, Maximum principles in differential equations, Prentice Hall, Englewood Cliffs, N.J., 1967.

28. F. Warner, Foundations of differential manifolds and Lie groups, Scott, Foresman and Co., Glenview, Il., 1971.

29. J. W. York, Covariant decompositions of symmetric tensors in the Theory of gravitation, Ann.

Inst. H. Poincaré Sect. A (N.S.) 21 (1974), 319-332.

30. K. Yoshida, Functional analysis, 3rd ed., Springer-Verlag, New York, 1971.

Department of Mathematics, University of TeXas, Austin, TeXas 78712 